\title{
Critical Fermi surfaces in generic dimensions arising from transverse gauge field interactions
}

\author{
Ipsita Mandal (1) \\ Faculty of Science and Technology, University of Stavanger, 4036 Stavanger, Norway \\ and Nordita, Roslagstullsbacken 23, SE-106 91 Stockholm, Sweden
}

(Received 18 June 2020; revised 7 November 2020; accepted 10 November 2020; published 23 November 2020; corrected 29 July 2021)

\begin{abstract}
We study critical Fermi surfaces in generic dimensions arising from coupling finite-density fermions with transverse gauge fields by applying a dimensional regularization scheme developed previously [Phys. Rev. B 92, 035141 (2015)]. We consider the cases of $U(1)$ and $U(1) \times U(1)$ transverse gauge couplings and extract the nature of the renormalization group (RG) flow fixed points as well as the critical scalings. Our analysis allows us to treat a critical Fermi surface of a generic dimension $m$ perturbatively in an expansion parameter $\epsilon=(2-m) /(m+1)$. One of our key results is that although the two-loop corrections do not alter the existence of an RG flow fixed line for certain $U(1) \times U(1)$ theories, which was identified earlier for $m=1$ at one-loop order, the third-order diagrams do. However, this fixed line feature is also obtained for $m>1$, where the answer is one-loop exact due to UV/IR mixing.
\end{abstract}

DOI: 10.1103/PhysRevResearch.2.043277

\section{INTRODUCTION}

Metallic states that lie beyond the framework of LandauFermi liquid theory are often dubbed non-Fermi liquids. A finite density of of nonrelativistic fermions coupled to a transverse $U(1)$ gauge field has been known as an example of a non-Fermi liquid. This model was first studied by Holstein, Norton, and Pincus [1], whose original motivation was to understand the effects of the electromagnetic field coupled to a metal. However, it was realized that the same field theory applies to the case of fermions coupled to Abelian or non-Abelian emergent gauge field(s) in various scenarios like certain quantum spin liquids [2], the normal state of cuprate superconductors [3-8], and the compressible quantum Hall systems at $1 / 2$-filling $[9,10]$. Such a fictitious transverse gauge field has the gauge coupling value of the order unity (and not the value 1/137 of the fine-structure constant of the electromagnetic forces) and consequently gives rise to a strongly correlated system. It is a theoretically challenging task to study such systems, and consequently there have been intensive efforts dedicated to building a framework to understand them [1,6,9-38]. They are also referred to as critical Fermi surface states, as the breakdown of the Fermi liquid theory is brought about by the interplay between the soft fluctuations of the Fermi surface and some gapless bosonic fluctuations. These bosonic degrees of freedom can be massless scalar bosons or the transverse components of gauge fields. A similar situation also arises in semimetals, where instead of a Fermi surface, there is a Fermi node

Published by the American Physical Society under the terms of the Creative Commons Attribution 4.0 International license. Further distribution of this work must maintain attribution to the author(s) and the published article's title, journal citation, and DOI. interacting with long-ranged (unscreened) Coulomb potential which gives rise to a non-Fermi liquid behavior [39-42]. Since the quasiparticles are destroyed, there is no obvious perturbative parameter in which one can carry out a controlled expansion, which would ultimately enable us to extract the universal properties.

In this paper, we consider the case when Fermi surfaces are coupled with emergent gauge fields $[9,10,23,25,26,43-46]$. This belongs to the category when the critical boson carries zero momentum, and the quasiparticles lose coherence across the entire Fermi surface. An example when the critical boson with zero momentum is a scalar is the Ising-nematic critical point $[15,17,19,28,30-33,47-60]$. There are complementary cases when the critical boson carries a finite momentum. Examples include the critical points involving a spin density wave (SDW), charge density wave (CDW) [20-22,29,34-36], and the FFLO order parameter [38].

An analytic approach $[28,30,38,61]$ to deal with non-Fermi liquid quantum critical points is through dimensional regularization, in which the co-dimension of the Fermi surface is increased in order to identify an upper critical dimension $d=$ $d_{c}$ and, subsequently, to calculate the critical exponents in a systematic expansion involving the parameter $\epsilon=d_{c}-d_{\text {phys }}$ (where $d_{\text {phys }}$ is the actual/physical dimension of the system). This approach is especially useful, as it allows one to deal with critical Fermi surfaces of a generic dimension $m$ [30,31], representing a system with physical dimensions $d=d_{\text {phys }}=$ $m+1$. The physical systems have $d_{\text {phys }}$ equal to two or three. Hence, $m$ is equal to one or two for the corresponding systems.

Another approach implements controlled approximation through dynamical tuning, involving an expansion in the inverse of the number $(N)$ of fermion flavors combined with a further expansion $\varepsilon=z_{b}-2$, where $z_{b}$ is the dynamical critical exponent of the boson field $[9,23]$. This amounts to modifying the kinetic term of a collective mode $[\phi(k)]$ from $k^{2}|\phi(k)|^{2}$ to $k^{1+\varepsilon}|\phi(k)|^{2}$. A drawback of this approach is that 
this modification of the kinetic term leads to nonanalyticities in the momentum space, which are equivalent to nonlocal hopping terms in real space. Hence in this paper, we will employ the former approach of dimensional regularization, which maintains locality in real space.

The earlier works considering generic values of $d$ and $m$ involved the Ising-nematic order parameter [30,31], which represents quantum critical metals near a Pomeranchuk transition, where the critical boson couples to antipodal patches with the same sign of coupling strength [19]. In contrast, a transverse gauge field couples to the two antipodal patches with opposite signs [18]. Here we will implement the dimensional regularization procedure to determine the low-energy scalings of an $m$-dimensional (with $m \geqslant 1$ ) Fermi surface coupled with one or more transverse gauge fields. First we will develop the formalism for a single $U(1)$ gauge field. Then we will extend it to the $U(1) \times U(1)$ case, which can describe a quantum phase transition between a Fermi liquid metal and an electrical insulator without any Fermi surface (deconfined Mott transition) or that between two metals that having Fermi surfaces with finite but different sizes on either side of the transition (deconfined metal-metal transition) [62].

The paper is organized as follows. In Sec. II we review the framework for applying a dimensional regularization scheme to access the non-Fermi liquid fixed points perturbatively, and apply it to the case of a single transverse gauge field. We also compute the renormalization of the $2 k_{F}$ scattering amplitude for the fermions and the scaling forms of some physical observables. In Sec. III we carry out the computations for the scenario of quantum critical transitions involving two different kinds of fermions charged differently under the action of two transverse gauge fields. We conclude with a summary and an outlook in Sec. IV. The details of the one-loop calculations are provided in the Appendix.

\section{MODEL INVOLVING A $U$ (1) TRANSVERSE GAUGE FIELD}

We first consider an $m$-dimensional Fermi surface, which is coupled to a $U(1)$ transverse gauge field $a$ in $d=(m+1)$ space dimensions. The setup is identical to that of Ref. [30]. We review it here for the sake of completeness. As in earlier works $[28,30,31]$, we want to characterize the resulting nonFermi liquids through the scaling properties of the fermionic and bosonic Green's functions. To do so, we focus on one point (say, $K^{*}$ ) of the Fermi surface at which the fermion Green's function is defined. The low-energy effective theory involves fermions which are primarily scattered along the tangential directions of the Fermi surface, mediated by the critical boson. We assume the presence of the inversion symmetry, which implies that the fermions near $K^{*}$ are most strongly coupled with fermions near the antipodal point $-K^{*}$, since their tangent spaces coincide. Hence we write a model including a closed Fermi surface divided into two halves centered at momenta $K^{*}$ and $-K^{*}$, respectively. The fermionic fields $\psi_{+, j}$ and $\psi_{-, j}$ represent the corresponding halves, as shown in Fig. 1(a). In this coordinate system, the minimal Euclidean action that captures the essential description of the

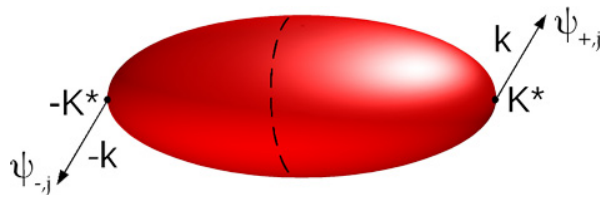

(a)
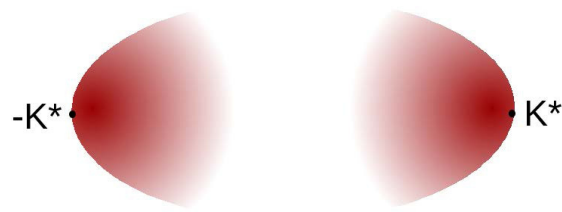

(b)

FIG. 1. (a) Schematic of a compact Fermi surface divided into two halves, which are centered at $K^{*}$ and $-K^{*}$, respectively. For the two halves, two separate fermionic fields $\left(\psi_{ \pm, j}\right)$ have been introduced. (b) In the effective action, a compact Fermi surface is approximated by two sheets of noncompact Fermi surfaces (approximated by parabolic dispersion to leading order). The momentum modes far away from $\pm K^{*}$ have been suppressed by using a momentum regularization.

low-energy physics is given by [18]

$$
\begin{aligned}
S= & \sum_{p= \pm} \sum_{j=1}^{N} \int d k \psi_{p, j}^{\dagger}(k)\left(i k_{0}+p k_{1}+\mathbf{L}_{(k)}^{2}\right) \psi_{p, j}(k) \\
& +\frac{1}{2} \int d k\left(k_{0}^{2}+k_{1}^{2}+\mathbf{L}_{(k)}^{2}\right) a^{\dagger}(k) a(k) \\
& +\frac{e}{\sqrt{N}} \sum_{p= \pm} p \sum_{j=1}^{N} \int d k d q a(q) \psi_{p, j}^{\dagger}(k+q) \psi_{p, j}(k),
\end{aligned}
$$

where $k=\left(k_{0}, k_{1}, \mathbf{L}_{(k)}\right) \quad$ is the $(d+1)$-dimensional energy-momentum vector with $d k \equiv \frac{d^{d+1} k}{(2 \pi)^{d+1}}$, and $e$ is the transverse gauge coupling. The fermion field $\psi_{+, j}\left(k_{0}, k_{i}\right) \quad\left(\psi_{-, j}\left(k_{0}, k_{i}\right)\right)$ with flavor $j=1,2, \ldots, N$, frequency $k_{0}$ and momentum $K_{i}^{*}+k_{i} \quad\left(-K_{i}^{*}+k_{i}\right)$ is represented by $\psi_{+, j}\left(k_{0}, k_{i}\right)\left(\psi_{-, j}\left(k_{0}, k_{i}\right)\right)$, with $1 \leqslant i \leqslant d$. The components $k_{1}$ and $\mathbf{L}_{(k)} \equiv\left(k_{2}, k_{3}, \ldots, k_{d}\right)$ represent the momentum components perpendicular and parallel to the Fermi surface at $\pm K^{*}$, respectively. We have rescaled the momentum such that the absolute value of the Fermi velocity and the quadratic curvature of the Fermi surface at $\pm K^{*}$ can be set to one. An actual physical situation can involve a Fermi surface of an arbitrary shape. Our physical setup allows us to include such a generic scenario as long as the Fermi surface is locally convex, as the coordinate has been set up with its origin at a particular small patch of the Fermi surface (see Fig. 2).

Due to the fact that the Fermi surface is locally parabolic, the scaling dimensions of $k_{1}$ and $\mathbf{L}_{(k)}$ are equal to 1 and $1 / 2$, respectively. For a generic convex Fermi surface, there can be cubic and higher-order terms in $\mathbf{L}_{(k)}$, but we can ignore them as they irrelevant in the renormalization group (RG) sense. Since we have a compact Fermi surface, the range 


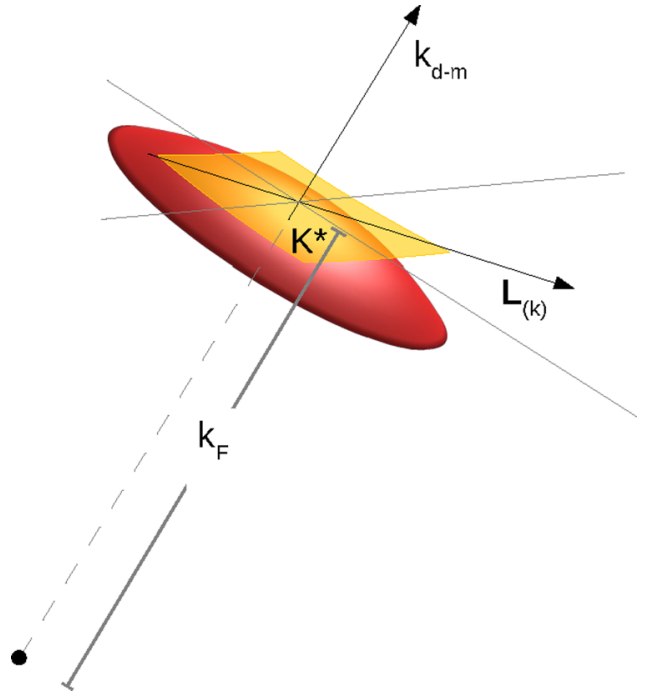

FIG. 2. The momentum coordinates on a patch of an $m$ dimensional convex Fermi surface.

of $\mathbf{L}_{(k)}$ in $\int d k$ is finite and is set by the size of the Fermi surface. This range is of the order of $\sqrt{k_{F}}$ in this coordinate system. To ensure this finite integration range, we will include an exponential cutoff $\exp \left\{-\frac{\mathbf{L}_{(k)}^{2}}{\mu \tilde{k}_{F}}\right\}$ while using the fermion Green's function in loop integrations, which will capture the compactness of the Fermi surface in a minimal way without including the details of the shape. This can be made explicit by including the inverse of this factor in the kinetic part of the fermion action.

In order to control the gauge coupling $e$ for a given $m$, we tune the co-dimension of the Fermi surface $[28,29,61]$ to determine the upper critical dimension $d=d_{c}$. To preserve the analyticity of the theory in momentum space (locality in real space) with general co-dimensions, we introduce the spinors [28,29]

$$
\Psi_{j}^{T}(k)=\left(\psi_{+, j}(k), \psi_{-, j}^{\dagger}(-k)\right) \text { and } \bar{\Psi}_{j} \equiv \Psi_{j}^{\dagger} \gamma_{0}
$$

and write an action that describes the $m$-dimensional Fermi surface embedded in a $d$-dimensional momentum space:

$$
\begin{aligned}
S= & \sum_{j} \int d k \bar{\Psi}_{j}(k) i\left(\boldsymbol{\Gamma} \cdot \mathbf{K}+\gamma_{d-m} \delta_{k}\right) \Psi_{j}(k) \exp \left(\frac{\mathbf{L}_{(k)}^{2}}{\mu \tilde{k}_{F}}\right) \\
& +\frac{1}{2} \int d k \mathbf{L}_{(k)}^{2} a^{\dagger}(k) a(k) \\
& +\frac{e \mu^{x / 2}}{\sqrt{N}} \sum_{j} \int d k d q a(q) \bar{\Psi}_{j}(k+q) \gamma_{0} \Psi_{j}(k) \\
x= & \frac{4+m-2 d}{2} .
\end{aligned}
$$

Here $\mathbf{K} \equiv\left(k_{0}, k_{1}, \ldots, k_{d-m-1}\right)$ includes the frequency and the first $(d-m-1)$ components of the $d$-dimensional momentum vector, $\mathbf{L}_{(k)} \equiv\left(k_{d-m+1}, \ldots, k_{d}\right)$ and $\delta_{k}=k_{d-m}+$ $\mathbf{L}_{(k)}^{2}$. In the $d$-dimensional momentum space, $k_{1}, \ldots, k_{d-m}$
$\left(\mathbf{L}_{(k)}\right)$ represent(s) the $(d-m)(m)$ directions perpendicular (tangential) to the Fermi surface. $\boldsymbol{\Gamma} \equiv\left(\gamma_{0}, \gamma_{1}, \ldots, \gamma_{d-m-1}\right)$ represents the gamma matrices associated with $\mathbf{K}$. Since we are interested in a value of co-dimension $1 \leqslant d-m \leqslant$ 2 , we consider only $2 \times 2$ gamma matrices with $\gamma_{0}=$ $\sigma_{y}, \gamma_{d-m}=\sigma_{x}$. In the quadratic action of the boson, only $\mathbf{L}_{(k)}^{2} a^{\dagger}(k) a(k)$ is kept, because $|\mathbf{K}|^{2}+k_{d-m}^{2}$ is irrelevant under the scaling where $k_{0}, k_{1}, \ldots, k_{d-m}$ have dimension 1 and $k_{d-m+1}, \ldots, k_{d}$ have dimension $1 / 2$. In the presence of the $(m+1)$-dimensional rotational symmetry, all components of $k_{d-m}, \ldots, k_{d}$ should be equivalent. The rotational symmetry of the bare fermion kinetic part in the $(d-m)$-dimensional space spanned by $\mathbf{K}$ components is destroyed by the coupling with the gauge boson, as the latter involves the $\gamma_{0}$ matrix. With this in mind, we will denote the extra (unphysical) codimensions by the vector $\tilde{\mathbf{K}}$ and the corresponding gamma matrices by $\tilde{\boldsymbol{\Gamma}}$.

Since the scaling dimension of the gauge coupling is equal to $x$, we have made $e$ dimensionless by using a mass scale $\mu$. We have also defined a dimensionless parameter for the Fermi momentum, $\tilde{k}_{F}=k_{F} / \mu$ using this mass scale. The spinor $\Psi_{j}$ exhibits an energy dispersion with two bands $E_{k}=$ $\pm \sqrt{\sum_{i=1}^{d-m-1} k_{i}^{2}+\delta_{k}^{2}}$, and this gives an $m$-dimensional Fermi surface embedded in the $d$-dimensional momentum space, defined by the $d-m$ equations: $k_{i}=0$ for $i=\{1, \ldots, d-$ $m-1\}$ and $k_{d-m}=-\mathbf{L}_{(k)}^{2}$. Basically, the extra $(d-m-1)$ directions are gapped out so that the Fermi surface reduces to a sphere $S^{m}$ [sphere in an $(m+1)$-dimensional Euclidean space] locally.

When we perform dimensional regularization, the theory implicitly has an ultraviolet (UV) cutoff for $\mathbf{K}$ and $k_{d-m}$, which we denote by $\Lambda$. It is natural to choose $\Lambda=\mu$, and the theory has two important dimensionless parameters: $e$ and $\tilde{k}_{F}=k_{F} / \Lambda$. If $k$ is the typical energy at which we probe the system, the limit of interest is $k \ll \Lambda \ll k_{F}$. This is because $\Lambda$ sets the largest energy (equivalently, momentum perpendicular to the Fermi surface) fermions can have, whereas $k_{F}$ sets the size of the Fermi surface. We will consider the RG flow generated by changing $\Lambda$ and requiring that lowenergy observables are independent of it. This is equivalent to a coarse-graining procedure of integrating out high-energy modes away from Fermi surface. Because the zero energy modes are not integrated out, $k_{F} / \Lambda$ keeps on increasing in the coarse-graining procedure. We treat $k_{F}$ as a dimensionful coupling constant that flows to infinity in the low-energy limit. Physically, this describes the fact that the size of the Fermi surface, measured in the unit of the thickness of the thin shell, around the Fermi surface diverges in the low-energy limit. This is illustrated in Fig. 1(a).

\section{A. Dimensional regularization}

To gain a controlled approximation of the physics of the critical Fermi surface, we fix $m$ and tune $d$ towards a critical dimension $d_{c}$, at which quantum corrections depend logarithmically on $\Lambda$ within the range $\Lambda \ll k_{F}$. In order to identify the value of $d_{c}$ as a function of $m$, we consider the one-loop quantum corrections. 


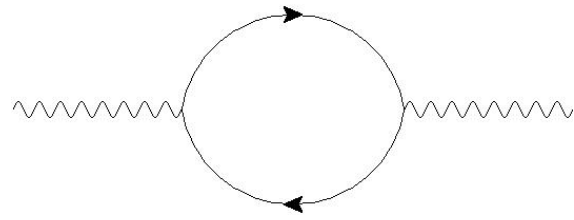

(a)

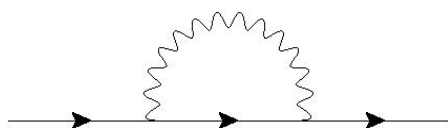

(b)

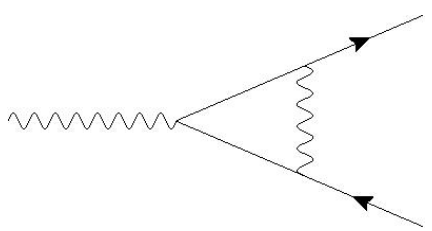

(c)

FIG. 3. The one-loop diagrams for (a) the boson self-energy, (b) the fermion self-energy, and (c) the vertex correction. Lines with arrows represent the bare fermion propagator, whereas wiggly lines in (b) and (c) represent the dressed boson propagator, which includes the one-loop self-energy in (a).

The bare propagator for fermions is given by

$$
G_{0}(k)=-i \frac{\boldsymbol{\Gamma} \cdot \mathbf{K}+\gamma_{d-m} \delta_{k}}{\mathbf{K}^{2}+\delta_{k}^{2}} \times \exp \left(-\frac{\mathbf{L}_{(k)}^{2}}{\mu \tilde{k}_{F}}\right) .
$$

Since the bare boson propagator is independent of $k_{0}, \ldots, k_{d-m}$, the loop integrations involving it are ill-defined, unless one resums a series of diagrams that provides a nontrivial dispersion along those directions. This amounts to rearranging the perturbative expansion such that the one-loop boson self-energy is included at the "zero"-th order. The dressed boson propagator includes the one-loop self-energy [see Fig. 3(a)] given by

$$
\begin{aligned}
& \Pi_{1}(k)=-e^{2} \mu^{x} \int d q \operatorname{Tr}\left[\gamma_{0} G_{0}(k+q) \gamma_{0} G_{0}(q)\right] \\
& =-\frac{\beta(d, m) e^{2} \mu^{x}\left(\mu \tilde{k}_{F}\right)^{\frac{m-1}{2}}}{\left|\mathbf{L}_{(k)}\right|} \\
& \quad \times\left[k_{0}^{2}+(m+1-d) \tilde{\mathbf{K}}^{2}\right]|\mathbf{K}|^{d-m-2},
\end{aligned}
$$

where

$$
\beta(d, m)=\frac{\pi^{\frac{4-d}{2}} \Gamma(d-m) \Gamma(m+1-d)}{2^{\frac{4 d-m-1}{2}} \Gamma^{2}\left(\frac{d-m+2}{2}\right) \Gamma\left(\frac{m+1-d}{2}\right)} .
$$

This expression is valid to the leading order in $k / k_{F}$, and for $|\mathbf{K}|^{2} /\left|\mathbf{L}_{(k)}\right|^{2}, \delta_{k}^{2} /\left|\mathbf{L}_{(k)}\right|^{2} \ll k_{F}$ [63]. We provide the details of computation for the expression of $\Pi_{1}(k)$ in Appendix A 1. For $m>1$, the boson self-energy diverges in the $k_{F} \rightarrow \infty$ limit. This is due to the fact that the Landau damping gets stronger for a system with a larger Fermi surface, as the boson can decay into particle-hole excitations that encompass the entire Fermi surface for $m>1$. This is in contrast with the case for $m=1$, where a low-energy boson with a given momentum can decay into particle-hole excitations only near the isolated patches whose tangent vectors are parallel to that momentum. Equation (5) is valid when there exists at least one direction that is tangential to the Fermi surface $(m \geqslant 1)$. Henceforth, we will use the dressed propagator

$$
D_{1}(k)=\frac{1}{\mathbf{L}_{(k)}^{2}-\Pi_{1}(k)}
$$

for any loop calculation.

The next step is to computed the one-loop fermion selfenergy $\Sigma_{1}(q)$, as shown in Fig. 3(b). Again, the details of the calculation are provided in Appendix A 2. This blows up logarithmically in $\Lambda$ at the critical dimension

$$
d_{c}(m)=m+\frac{3}{m+1} .
$$

The physical dimension is given by $d=d_{c}(m)-\epsilon$. In the dimensional regularization scheme, the logarithmic divergence in $\Lambda$ turns into a pole in $\frac{1}{\epsilon}$ :

$$
\Sigma_{1}(k)=-\frac{i e^{\frac{2(m+1)}{3}}\left[u_{0} \gamma_{0} k_{0}+u_{1}(\tilde{\boldsymbol{\Gamma}} \cdot \tilde{\mathbf{K}})\right]}{N \tilde{k}_{F}^{\frac{(m-1)(2-m)}{6}} \epsilon}+\text { finite terms }
$$

to the leading order in $k / k_{F}$, where $u_{0}, u_{1} \geqslant 0$. For the cases of interest, we have computed these coefficients numerically to obtain

$$
\left\{\begin{array}{ll}
u_{0}=0.0201044, \quad u_{1}=1.85988 & \text { for } m=1 \\
u_{0}=u_{1}=0.0229392 & \text { for } m=2
\end{array} .\right.
$$

The one-loop vertex correction in Fig. 3(c) is nondivergent and hence does not contribute to the RG flows. This is to be contrasted with the Ising-nematic case where it is guaranteed to vanish due to a Ward identity [28].

We can vary the dimension of Fermi surface from $m=1$ to $m=2$ while keeping $\epsilon$ small, thus providing a controlled description for any $m$ between 1 and 2. For a given $m$, we tune $d$ such that $\epsilon=d_{c}(m)-d$ is small. To remove the UV divergences in the $\epsilon \rightarrow 0$ limit, we add counterterms using the minimal subtraction scheme. The counterterms take the same form as the original local action:

$$
\begin{aligned}
S_{C T}= & \sum_{j} \int d k \bar{\Psi}_{j}(k) i\left(A_{0} \gamma_{0} k_{0}+A_{1} \tilde{\boldsymbol{\Gamma}} \cdot \tilde{\mathbf{K}}+A_{2} \gamma_{d-m} \delta_{k}\right) \Psi_{j}(k) \exp \left(\frac{\mathbf{L}_{(k)}^{2}}{\mu \tilde{k}_{F}}\right) \\
& +\frac{A_{3}}{2} \int d k \mathbf{L}_{(k)}^{2} a^{\dagger}(k) a(k)+A_{4} \frac{e \mu^{x / 2}}{\sqrt{N}} \sum_{j} \int d k d q a(q) \bar{\Psi}_{j}(k+q) \gamma_{0} \Psi_{j}(k),
\end{aligned}
$$


where

$$
A_{\zeta}=\sum_{\lambda=1}^{\infty} \frac{Z_{\zeta}^{(\lambda)}\left(e, \tilde{k}_{F}\right)}{\epsilon^{\lambda}} \text { with } \zeta=0,1,2,3,4
$$

In the mass-independent minimal subtraction scheme, these coefficients depend only on the scaled coupling $e$ and the scaled Fermi momentum $\tilde{k}_{F}$. As discussed earlier, we expect $\tilde{k}_{F}$ to act as another coupling for $m>1$, and hence it must be included in the RG flow equations. The coefficients can be further expanded in the number of loops modulo the one-loop self-energy of boson, which is already included in Eq. (7).
Note that the $(d-m-1)$-dimensional rotational invariance in the space perpendicular to the Fermi surface guarantees that each term in $\tilde{\boldsymbol{\Gamma}} \cdot \tilde{\mathbf{K}}$ is renormalized in the same way. Similarly, the sliding symmetry along the Fermi surface guarantees that the form of $\delta_{k}$ is preserved. However, $A_{0}, A_{1}$, and $A_{2}$ are in general different due to a lack of the full rotational symmetry in the $(d+1)$-dimensional spacetime. Note the difference from the Ising-nematic case, where we had $A_{0}=A_{1}$, as the rotational symmetry there involved the full $(d-m)$ dimensional subspace.

Adding the counterterms to the original action, we obtain the renormalized action which gives the finite quantum effective action:

$$
\begin{aligned}
S_{\text {ren }}= & \sum_{j} \int d k^{B} \bar{\Psi}_{j}^{B}\left(k^{B}\right) i\left(\gamma_{0} k_{0}^{B}+\tilde{\boldsymbol{\Gamma}} \cdot \tilde{\mathbf{K}}^{B}+\gamma_{d-m} \delta_{k^{B}}\right) \Psi_{j}^{B}\left(k^{B}\right) \exp \left(\frac{\mathbf{L}_{\left(k^{B}\right)}^{2}}{k_{F^{B}}}\right)+\frac{1}{2} \int d k^{B} \mathbf{L}_{\left(k^{B}\right)}^{2} a^{B^{\dagger}}\left(k^{B}\right) a^{B}\left(k^{B}\right) \\
& +\frac{e^{B}}{\sqrt{N}} \sum_{j} \int d k^{B} d q^{B} a^{B}\left(q^{B}\right) \bar{\Psi}_{j}^{B}\left(k^{B}+q^{B}\right) \gamma_{0} \Psi_{j}^{B}\left(k^{B}\right),
\end{aligned}
$$

where

$$
\begin{aligned}
k_{0}^{B} & =\frac{Z_{0}}{Z_{2}} k_{0}, \quad \tilde{\mathbf{K}}^{B}=\frac{Z_{1}}{Z_{2}} \tilde{\mathbf{K}}, \quad k_{d-m}^{B}=k_{d-m}, \\
\mathbf{L}_{\left(k^{B}\right)} & =\mathbf{L}_{(k)}, \quad \Psi_{j}^{B}\left(k^{B}\right)=Z_{\Psi}^{\frac{1}{2}} \Psi_{j}(k), \\
a^{B}\left(k^{B}\right) & =Z_{a}^{\frac{1}{2}} a(k), \quad k_{F}^{B}=k_{F}=\mu \tilde{k}_{F}, \\
Z_{\Psi} & =\frac{Z_{2}^{d-m+1}}{Z_{0} Z_{1}^{d-m-1}}, \quad Z_{a}=\frac{Z_{3} Z_{2}^{d-m}}{Z_{0} Z_{1}^{d-m-1}}, \\
e^{B} & =Z_{e} e \mu^{\frac{x}{2}}, \quad Z_{e}=\frac{Z_{4} Z_{2}^{\frac{d-m}{2}-1}}{\sqrt{Z_{0} Z_{3}} Z_{1}^{\frac{d-m-1}{2}}} .
\end{aligned}
$$

Here

$$
Z_{\zeta}=1+A_{\zeta} .
$$

The superscript " $\mathrm{B}$ " denotes the bare fields, couplings, and momenta. In Eq. (13) there is a freedom to change the renormalizations of the fields and the renormalization of momentum without affecting the action. Here we fix the freedom by requiring that $\delta_{k^{B}}=\delta_{k}$. This amounts to measuring scaling dimensions of all other quantities relative to that of $\delta_{k}$.

Let $z$ be the dynamical critical exponent, $\tilde{z}$ be the critical exponent along the extra spatial dimensions, $\beta_{e}$ be the beta function for the coupling $e, \beta_{k_{F}}$ be the beta function for $\tilde{k}_{F}$, and $\eta_{\Psi}\left(\eta_{\phi}\right)$ be the anomalous dimension for the fermions (gauge boson). These are explicitly given by

$$
\begin{aligned}
& z=1+\frac{\partial \ln \left(Z_{0}\right)}{\partial \ln \mu}, \quad \tilde{z}=1+\frac{\partial \ln \left(Z_{1}\right)}{\partial \ln \mu}, \quad \eta_{\Psi}=\frac{1}{2} \frac{\partial \ln Z_{\Psi}}{\partial \ln \mu}, \\
& \eta_{a}=\frac{1}{2} \frac{\partial \ln Z_{a}}{\partial \ln \mu}, \quad \beta_{k_{F}}\left(\tilde{k}_{F}\right)=\frac{\partial \tilde{k}_{F}}{\partial \ln \mu}, \quad \beta_{e}=\frac{\partial e}{\partial \ln \mu} . \quad \text { (16) }
\end{aligned}
$$

In the $\epsilon \rightarrow 0$ limit, we require solutions of the form

$$
\begin{aligned}
& z=z^{(0)}, \quad \tilde{z}=\tilde{z}^{(0)}, \\
& \eta_{\Psi}=\eta_{\Psi}^{(0)}+\eta_{\Psi}^{(1)} \epsilon, \quad \eta_{a}=\eta_{a}^{(0)}+\eta_{a}^{(1)} \epsilon .
\end{aligned}
$$

\section{B. RG flows at one-loop order}

To one-loop order, the counterterms are given by $Z_{\zeta}=1+$ $\frac{Z_{\xi}^{(1)}}{\epsilon}$. Collecting all the results, we find that only

$$
Z_{0}^{(1)}=-\frac{u_{0} \tilde{e}}{N} \text { and } Z_{1}^{(1)}=-\frac{u_{1} \tilde{e}}{N}
$$

are nonzero, where

$$
\tilde{e}=\frac{e^{\frac{2(m+1)}{3}}}{\tilde{k}_{F}^{\frac{(m-1)(2-m)}{6}}} .
$$

Then the one-loop beta functions, that dictate the flow of $\tilde{k}_{F}$ and $e$ with the increasing energy scale $\mu$, are given by

$$
\begin{aligned}
\beta_{k_{F}} & =-\tilde{k}_{F}, \quad(1-z) Z_{0}=-\beta_{e} \frac{\partial Z_{0}}{\partial e}+\tilde{k}_{F} \frac{\partial Z_{0}}{\partial \tilde{k}_{F}}, \\
(1-\tilde{z}) Z_{1} & =-\beta_{e} \frac{\partial Z_{1}}{\partial e}+\tilde{k}_{F} \frac{\partial Z_{1}}{\partial \tilde{k}_{F}}, \\
\beta_{e} & =-\frac{\epsilon+\frac{2-m}{m+1}(1-\tilde{z})+1-z}{2} e, \\
\eta_{\Psi} & =\eta_{a}=\frac{1-z+(1-\tilde{z})(d-m-1)}{2} .
\end{aligned}
$$

Solving these equations using the required form outlined in Eq. (17), we get

$$
\begin{aligned}
z & =1-\frac{(m+1) u_{0} \tilde{e}}{3 N+(m+1) u_{1} \tilde{e}}, \\
\tilde{z} & =1-\frac{(m+1) u_{1} \tilde{e}}{3 N+(m+1) u_{1} \tilde{e}}, \\
-\frac{\beta_{e}}{e} & =\frac{\epsilon}{2}+\frac{(m-1)(2-m)}{4(m+1)}-\frac{(m+1) u_{0}+(2-m) u_{1}}{6 N} \tilde{e} .
\end{aligned}
$$

The first term indicates that $e$ remains strictly relevant in the infrared (IR) at $d=d_{c}(m)$ for $1<m<2$. However, the 
second term implies that the higher-order corrections are controlled not by $e$, but by an effective coupling $\tilde{e}$. Indeed, the scaling dimension of $\tilde{e}$ vanishes at $d_{c}$ for $1 \leqslant m \leqslant 2$. The beta function of this effective coupling is given by

$$
\frac{\beta_{\tilde{e}}}{\tilde{e}}=-\frac{(m+1) \epsilon}{3}+\frac{(m+1)\left[(m+1) u_{0}+(2-m) u_{1}\right]}{9 N} \tilde{e} .
$$

The interacting fixed point is obtained from $\beta_{\tilde{e}}=0$ and takes the form

$$
\tilde{e}^{*}=\frac{3 N \epsilon}{(m+1) u_{0}+(2-m) u_{1}}+O\left(\epsilon^{2}\right) .
$$

It can be checked that this is an IR stable fixed point by computing the first derivative of $\beta_{\tilde{e}}$. The critical exponents at this stable fixed point are given by

$$
\begin{aligned}
z^{*} & =1+\frac{(m+1) u_{1} \epsilon}{(m+1) u_{0}+(2-m) u_{1}}, \\
\tilde{z}^{*} & =1+\frac{(m+1) u_{0} \epsilon}{(m+1) u_{0}+(2-m) u_{1}}, \\
\eta_{\Psi}^{*} & =\eta_{a}^{*}=-\frac{\epsilon}{2} .
\end{aligned}
$$

\section{Higher-loop corrections}

We will now discuss the implications of the higher-loop corrections, without actually computing the Feynman diagrams. For $m>1$, we expect a nontrivial UV/IR mixing to be present, as was found in Refs. [30,31], which makes the results one-loop exact. In other words, all higher-loop corrections would vanish for $m>1$ in the limit $k_{F} \rightarrow 0$, due to suppression of the results by positive powers of $k_{F}$. For $m=1$, we will use the arguments and results of Ref. [28] to assume a generic form of the corrections coming from two-loop diagrams. Henceforth, we will just focus on $m=1$ in this subsection.

The two-loop diagrams for the boson self-energy have been shown in Fig. 4. The value should turn out to be UV finite and hence will renormalize the factor $\beta\left(\frac{5}{2}, 1\right)$ [see Eq. (6)] by a finite amount $\beta_{2}=\frac{\kappa \tilde{e}}{N}$, where $\kappa$ is a finite number. Then the bosonic propagator at this order will take the form

$$
D_{2}(q)=\frac{1}{\mathbf{L}_{(q)}^{2}+\frac{\left[\beta\left(\frac{5}{2}, 2\right)+\frac{\kappa \tilde{e}}{N}\right] e^{2} \mu^{\epsilon}}{\left|\mathbf{L}_{(q)}\right|} \times \frac{k_{0}^{2}+\left(\epsilon-\frac{1}{2}\right) \tilde{\mathbf{K}}^{2}}{|\mathbf{K}|^{\frac{1}{2}+\epsilon}} .}
$$

From this, the fermion self-energy now receives a correction

$$
\begin{aligned}
\Sigma_{2}^{(1)}(k) & =\left\{\left[\frac{\beta\left(\frac{5}{2}, 2\right)}{\beta\left(\frac{5}{2}, 2\right)+\frac{\kappa \tilde{e}}{N}}\right]^{\frac{1}{3}}-1\right\} \Sigma_{1}(k) \\
& =-\frac{\kappa \tilde{e}}{3 N \beta\left(\frac{5}{2}, 2\right)} \Sigma_{1}(k)+\text { finite terms. }
\end{aligned}
$$

Now the two-loop fermion self-energy diagrams (see Fig. 5), after taking into account the counterterms obtained

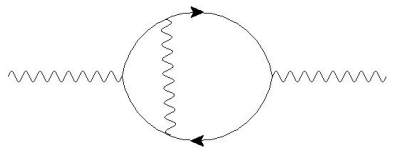

(a)

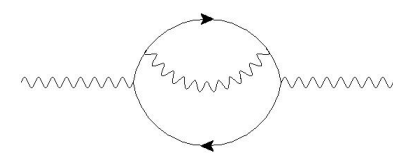

(b)

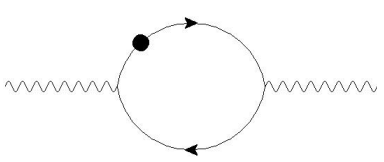

(d)

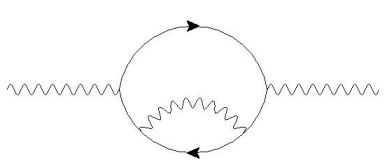

(c)

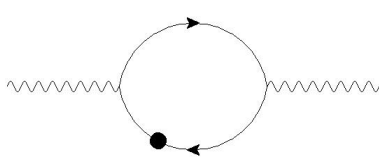

(e)
FIG. 4. The two-loop diagrams contributing to the boson selfenergy. Each black dot denotes the one-loop counterterm for the fermion self-energy.

from one-loop corrections, take the form

$$
\begin{aligned}
\Sigma_{2}^{(2)}(k)= & -\frac{i \tilde{e}^{2}\left[\tilde{v}_{0} \gamma_{0} q_{0}+\tilde{v}_{1}(\tilde{\boldsymbol{\Gamma}} \cdot \tilde{\mathbf{Q}})+w \gamma_{d-1} \delta_{k}\right]}{N^{2} \epsilon} \\
& + \text { finite terms. }
\end{aligned}
$$

Adding the two, generically the total two-loop fermion selfenergy can be written as

$$
\begin{aligned}
\Sigma_{2}^{\mathrm{tot}}(k)= & -\frac{i \tilde{e}^{2}\left[v_{0} \gamma_{0} q_{0}+v_{1}(\tilde{\boldsymbol{\Gamma}} \cdot \tilde{\mathbf{Q}})+w \gamma_{d-1} \delta_{k}\right]}{N^{2} \epsilon} \\
& + \text { finite terms, }
\end{aligned}
$$

where $v_{0}=u_{0}+\tilde{v}_{0}$ and $v_{1}=u_{1}+\tilde{v}_{1}$.

There will also be a divergent vertex correction (see Fig. 6), which will lead to a nonzero $Z_{4}^{(1)}$ of the form $-\frac{\tilde{e}^{2} \mu^{\frac{4 \epsilon}{3}} y}{N^{2}}$. All

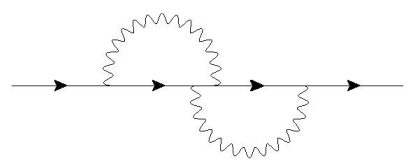

(a)

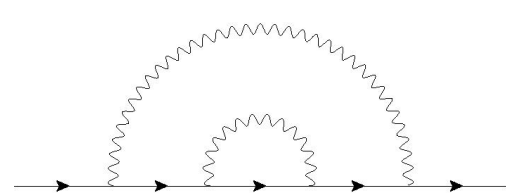

(b)

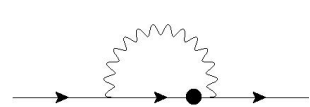

(c)
FIG. 5. The two-loop diagrams contributing to the fermion selfenergy. Each black dot denotes the one-loop counterterm for the fermion self-energy. 
these now lead to the nonzero coefficients:

$$
\begin{aligned}
& Z_{0}^{(1)}=-\frac{u_{0} \tilde{e}}{N}-\frac{v_{0} \tilde{e}^{2}}{N^{2}}, \quad Z_{1}^{(1)}=-\frac{u_{1} \tilde{e}}{N}-\frac{v_{0} \tilde{e}^{2}}{N^{2}}, \\
& Z_{2}^{(1)}=-\frac{w \tilde{e}^{2}}{N^{2}}, \quad Z_{4}^{(1)}=-\frac{y \tilde{e}^{2}}{N^{2}},
\end{aligned}
$$

resulting in

$$
\frac{\beta_{\tilde{e}}}{\tilde{e}}=-\frac{2\left(2 u_{1} \tilde{e}+3 N\right) \epsilon}{9 N}+\frac{2\left[2 \tilde{e}\left(2 u_{0} u_{1}+u_{1}^{2}+6 v_{0}+3 v_{1}-9 w\right)+3 N\left(2 u_{0}+u_{1}\right)\right] \tilde{e}}{27 N^{2}} .
$$

At the fixed point, we now have

$$
\frac{\tilde{e}^{*}}{N}=\frac{3 \epsilon}{2 u_{0}+u_{1}}-\frac{18\left(2 v_{0}+v_{1}-3 w\right)}{\left(2 u_{0}+u_{1}\right)^{3}} \epsilon^{2}+O\left(\epsilon^{3}\right)
$$

This shows that the nature of the stable non-Fermi liquid fixed point remains unchanged, although its location (as well as any critical scaling) gets corrected by one higher power of $\epsilon$.

\section{Renormalization of the $2 \boldsymbol{k}_{F}$ scattering amplitude}

In order to examine how the back-scattering is affected by the interactions with the gauge bosons in the non-Fermi liquid state, we consider an operator which carries momentum $2 k_{F}$ as follows:

$$
\begin{aligned}
S_{2 k_{F}}= & -2 g_{2 k_{F}} \mu \sum_{j} \int d k\left[\left(\psi_{+, j}^{\dagger}(k) \psi_{-, j}(k)\right.\right. \\
& \left.+\psi_{-, j}^{\dagger}(k) \psi_{+, j}(k)\right] \\
= & i g_{2 k_{F}} \mu \int d k\left[\Psi^{T}(k) \gamma_{0} \Psi(-k)+\bar{\Psi}(k) \gamma_{0} \bar{\Psi}^{T}(-k)\right],
\end{aligned}
$$

where $g_{2 k_{F}}$ is the source. To cancel UV divergences, we need to add a counterterm of the form

$$
\begin{aligned}
S_{2 k_{F}}^{C T}= & i g_{2 k_{F}} \mu\left(Z_{2 k_{F}}-1\right) \\
& \times \int d k\left[\Psi^{T}(k) \gamma_{0} \Psi(-k)+\bar{\Psi}(k) \gamma_{0} \bar{\Psi}^{T}(-k)\right],
\end{aligned}
$$

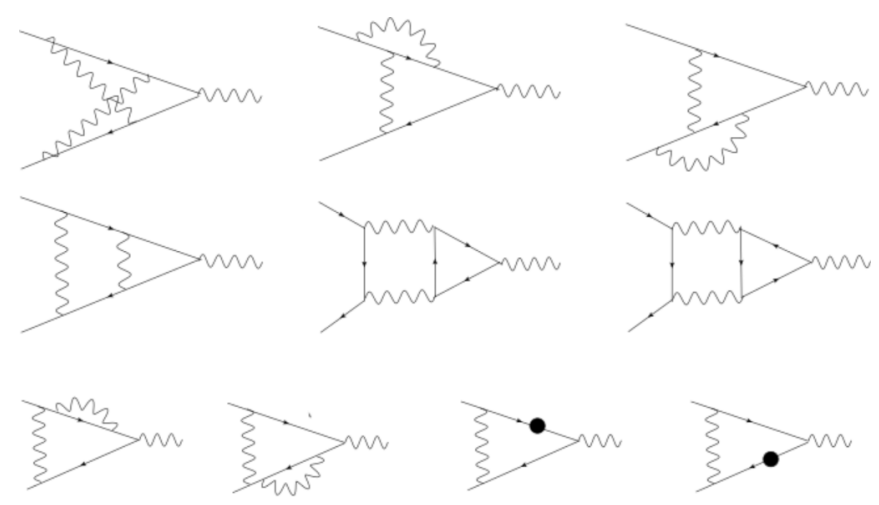

FIG. 6. The two-loop diagrams contributing to the vertex correction. Each black dot denotes the one-loop counterterm for the fermion self-energy. which renormalizes the insertion into

$$
\begin{aligned}
S_{2 k_{F}}^{\mathrm{ren}}= & i g_{2 k_{F}}^{B} \int d k^{B}\left\{\left[\Psi^{B}(k)\right]^{T} \gamma_{0} \Psi^{B}(-k)\right. \\
& \left.+\bar{\Psi}^{B}(k) \gamma_{0}\left[\bar{\Psi}^{B}(-k)\right]^{T}\right\}, \\
g_{2 k_{F}}^{B}= & Z_{g} g_{2 k_{F}} \mu, \quad Z_{2 k_{F}}=Z_{g} Z_{2} .
\end{aligned}
$$

Here

$$
Z_{2 k_{F}}=1+\frac{Z_{2 k_{F}}^{(1)}}{\epsilon}
$$

to one-loop order. The loop calculations involving the diagrams in Fig. 7 have been shown in details in Appendix A 4, which lead to

$$
Z_{2 k_{F}}^{(1)}=\left\{\begin{array}{ll}
-\frac{0.0774559 \tilde{e}}{N} & \text { for } m=1 \\
0 & \text { for } m=2
\end{array} .\right.
$$

This gives the beta function for $g_{2 k_{F}}$ as

$$
\beta_{g}=-g_{2 k_{F}}\left(1-\eta_{g}\right),
$$

with anomalous dimension $\eta_{g}=-\frac{2 \tilde{e} u_{g}}{3 N}$, where $u_{g}=$ 0.0774559 for $m=1$. The negative value of $\eta_{g}$ shows that the $2 k_{F}$ scattering amplitude is enhanced by fluctuations of the transverse gauge field. This is in contrast with the behavior computed in the case of in the Ising-nematic quantum criticality, where the $2 k_{F}$ scattering amplitude is suppressed [28] in the presence of the Ising-nematic critical bosons in $d_{\text {phys }}=2$. Note that this scattering amplitude can also be interpreted as an instability in the charge density wave (CDW) channel, which therefore (due to its negative anomalous dimension) turns out to be a serious competitor for the transverse gauge field criticality for $m=1$.

\section{E. Thermodynamic quantities}

The scaling of thermodynamic quantities are different from observables which are local in momentum space. This is because all low-energy modes near the Fermi surface contribute

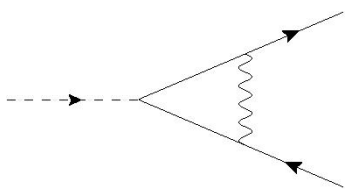

(a)

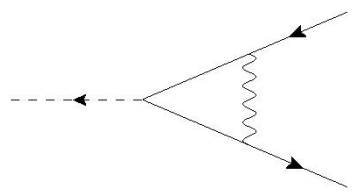

(b)
FIG. 7. The one-loop diagrams contributing to the $2 k_{F}$ scattering amplitude. 
to the thermodynamic responses. Here we will outline the expectations of a general scaling analysis.

In this paper, the momentum components $k_{d-m}$ and $\mathbf{L}_{(k)}$ have scaling dimensions one, $k_{0}$ has scaling dimension $z$, and the $d_{c}(m)-m-\epsilon-1=\frac{2-m}{m+1}-\epsilon$ momentum components with Dirac dispersion have scaling dimension $\tilde{z}$. Note that for Ising-nematic critical point $\tilde{z}=z$.

In order to examine the scaling behavior of thermodynamic quantities, we consider the free energy density at finite temperature $T$. In a system with $d_{\text {phys }}=m+1$ spatial dimensions, fermionic dynamical critical exponent $z$, and $\frac{2-m}{m+1}-\epsilon$ auxiliary dimensions with critical exponent $\tilde{z}$, the free energy density $F(T)$ has the scaling dimension $[F(T)]=d_{\text {phys }}+z+$ $\left(\frac{2-m}{m+1}-\epsilon\right) \tilde{z}$, if it were independent of any UV cutoff scale. However, when a critical boson couples with fermions on all parts of the Fermi surface, the entire Fermi surface becomes hot. As a result, we expect a hyperscaling violation, such that the the singular part of the free energy density depends on the size of the Fermi surface [32,64]. The largest momentum along the $\mathbf{L}_{(k)}$ direction is set by the Fermi momentum $k_{F}$, and hence the free energy density should have the scaling form

$$
F(T) \sim k_{F}^{m / 2} T^{1+\frac{d_{\text {phys }}-m}{z}+\frac{\left(\frac{2-m}{m+1}-\epsilon\right) \tilde{z}}{z}} \sim k_{F}^{m / 2} T^{1+\frac{1+\left(\frac{2-m}{m+1}-\epsilon\right) \tilde{z}}{z}}
$$

in the presence of an $m$-dimensional Fermi surface, with an effective scaling dimension $[F(T)]_{\mathrm{eff}}=1+z+\left(\frac{2-m}{m+1}-\epsilon\right) \tilde{z}$. From this scaling form, we can extract the temperature dependence of various observables within the quantum critical region. For example, the specific heat should scales as $C \propto$ $T^{\frac{1+\left(\frac{2-m}{m+1}-\epsilon\right) \tilde{z}}{z}}$.

The current operator is given by $J(T)=\frac{\delta F(T)}{\delta A}$, where $A$ is the vector potential with scaling dimension one. Hence, it should have the scaling form

$$
J(T) \sim k_{F}^{m / 2} T^{1+\frac{\left(\frac{2-m}{m+1}-\epsilon\right) \tilde{z}}{z},}
$$

with an effective scaling dimension $[J(T)]_{\mathrm{eff}}=z+$ $\left(\frac{2-m}{m+1}-\epsilon\right) \tilde{z}$. Then using the Kubo formula, we can infer that the effective scaling dimension of the optical conductivity is

$$
\begin{aligned}
& {[\sigma(\omega)]_{\mathrm{eff}}} \\
& \quad=2[J(T)]_{\mathrm{eff}}-z-[\text { volume in } k \text {-space }]_{\mathrm{eff}} \\
& \quad=2 z+2\left(\frac{2-m}{m+1}-\epsilon\right) \tilde{z}-z-z-1-\left(\frac{2-m}{m+1}-\epsilon\right) \tilde{z} \\
& \quad=-1+\left(\frac{2-m}{m+1}-\epsilon\right) \tilde{z},
\end{aligned}
$$

leading to the scaling form

$$
[\sigma(\omega \gg T)] \propto \omega^{-\frac{1}{z}+\frac{\left(\frac{2-m}{m+1}-\epsilon\right) \tilde{z}}{z},}
$$

where $\omega$ is the frequency of the applied ac electric field.

\section{MODEL INVOLVING TWO $U$ (1) TRANSVERSE GAUGE FIELDS}

In this section, we consider the $m$-dimensional Fermi surfaces of two different kinds of fermions (denoted by subscripts 1 and 2) coupled to two U(1) gauge fields, $a_{c}$ and $a_{s}$, in the context of deconfined Mott transition and deconfined metal-metal transition studied in Ref. [62] (for $m=1$ ). The theoretical motivation of Ref. [62] was to study a distinct class of quantum phase transitions between a Fermi liquid and a Mott insulator [65], or between two metals that have Fermi surfaces with finite but different sizes on either side of the transition $[66,67]$. These have been dubbed by the authors as deconfined Mott transition (DMT), and deconfined metal-metal transition $\left(\mathrm{DM}^{2} \mathrm{~T}\right)$, respectively. These problems can be formulated using a fictitious or emergent $U(2)$ gauge field, but the authors showed that this non-Abelian gauge field is "quasi-Abelianized" such that a related $U(1) \times U(1)$ gauge theory can capture many essential features. In this $U(1) \times U(1)$ gauge theory, the fermion fields $\psi_{1, \pm, j}$ and $\psi_{2, \pm, j}$ carry negative charges under the even $\left(a_{c}+a_{s}\right)$ and odd $\left(a_{c}-a_{s}\right)$ combinations of the gauge fields. We revisit this problem using our dimensional regularization scheme because, using this technique, we can study this system in generic dimensions and also perform higher-loop diagrams giving order by order corrections in $\epsilon$.

The action takes the form

$$
\begin{aligned}
S= & \sum_{\alpha=1,2} \sum_{p= \pm} \sum_{j=1}^{N} \int d k \psi_{\alpha, p, j}^{\dagger}(k)\left(i k_{0}+p k_{d-m}+\mathbf{L}_{(k)}^{2}\right) \psi_{\alpha, p, j}(k)+\frac{1}{2} \int d k \mathbf{L}_{(k)}^{2}\left[a_{c}^{\dagger}(k) a_{c}(k)+a_{s}^{\dagger}(k) a_{s}(k)\right] \\
& +\sum_{\alpha=1,2} \sum_{p= \pm} p \sum_{j=1}^{N} \int d k d q\left[\frac{(-1)^{\alpha} e_{s}}{\sqrt{N}} a_{s}(q) \psi_{\alpha, p, j}^{\dagger}(k+q) \psi_{\alpha, p, j}(k)-\frac{e_{c}}{\sqrt{N}} a_{c}(q) \psi_{\alpha, p, j}^{\dagger}(k+q) \psi_{\alpha, p, j}(k)\right],
\end{aligned}
$$

where $e_{c}$ and $e_{s}$ denote the gauge couplings for the gauge fields $a_{c}$ and $a_{s}$ respectively. We will perform dimensional regularization on this action and determine the RG fixed points. Our formalism allows us to extend the discussion beyond $m=1$ and also to easily compute higher-loop corrections.

\section{A. Dimensional regularization}

Proceeding as in the single transverse gauge field case, we add artificial co-dimensions for dimensional regularization after introducing the two-component spinors:

$$
\begin{aligned}
& \Psi_{\alpha, j}^{T}(k)=\left(\psi_{\alpha,+, j}(k), \psi_{\alpha,-, j}^{\dagger}(-k)\right) \text { and } \bar{\Psi}_{\alpha, j} \equiv \Psi_{\alpha, j}^{\dagger} \gamma_{0}, \\
& \text { with } \alpha=1,2 \text {. }
\end{aligned}
$$

The dressed gauge boson propagators include the one-loop self-energies given by

$$
\begin{aligned}
\Pi_{1}^{c}(k)= & -\frac{\beta(d, m) e_{c}^{2} \mu^{x}\left(\mu \tilde{k}_{F}\right)^{\frac{m-1}{2}}}{\left|\mathbf{L}_{(q)}\right|} \\
& \times\left[k_{0}^{2}+(m+1-d) \tilde{\mathbf{K}}^{2}\right]|\mathbf{K}|^{d-m-2}
\end{aligned}
$$


and

$$
\Pi_{1}^{s}(k)=-\frac{\beta(d, m) e_{s}^{2} \mu^{x}\left(\mu \tilde{k}_{F}\right)^{\frac{m-1}{2}}}{\left|\mathbf{L}_{(q)}\right|}\left[k_{0}^{2}+(m+1-d) \tilde{\mathbf{K}}^{2}\right]|\mathbf{K}|^{d-m-2},
$$

for the $a_{c}$ and $a_{s}$ gauge fields, respectively. This implies that the one-loop fermion self-energy for both $\Psi_{1, j}$ and $\Psi_{2, j}$ now takes the form

$$
\Sigma_{1}(q)=-\frac{i\left(e_{c}^{\frac{2(m+1)}{3}}+e_{s}^{\frac{2(m+1)}{3}}\right)}{N \tilde{k}_{F}^{\frac{(m-1)(2-m)}{6}}} \frac{u_{0} \gamma_{0} q_{0}+u_{1}(\tilde{\boldsymbol{\Gamma}} \cdot \tilde{\mathbf{Q}})}{\epsilon}+\text { finite terms, }
$$

with the critical dimension $d_{c}=\left(m+\frac{3}{m+1}\right), u_{0}$ and $u_{1}$ [see Eq. (10)] having the same values as for the $U(1)$ case.

The counterterms take the same form as the original local action:

$$
\begin{aligned}
S_{C T}= & \sum_{\alpha, j} \int d k \bar{\Psi}_{\alpha, j}(k) i\left(A_{0} \gamma_{0} k_{0}+A_{1} \tilde{\boldsymbol{\Gamma}} \cdot \tilde{\mathbf{K}}+A_{2} \gamma_{d-m} \delta_{k}\right) \Psi_{\alpha, j}(k) \exp \left(\frac{\mathbf{L}_{(k)}^{2}}{\mu \tilde{k}_{F}}\right)+\frac{A_{3_{s}}}{2} \int d k \mathbf{L}_{(k)}^{2} a_{s}^{\dagger}(k) a_{s}(k) \\
& +\frac{A_{3_{c}}}{2} \int d k \mathbf{L}_{(k)}^{2} a_{c}^{\dagger}(k) a_{c}(k)-A_{4_{c}} \frac{e_{c} \mu^{x / 2}}{\sqrt{N}} \sum_{\alpha, j} \int d k d q a_{c}(q) \bar{\Psi}_{\alpha, j}(k+q) \gamma_{0} \Psi_{\alpha, j}(k) \\
& +A_{4_{s}} \frac{e_{s} \mu^{x / 2}}{\sqrt{N}} \sum_{\alpha, j}(-1)^{\alpha} \int \frac{d^{d+1} k d^{d+1} q}{(2 \pi)^{2 d+2}} a_{s}(q) \bar{\Psi}_{\alpha, j}(k+q) \gamma_{0} \Psi_{\alpha, j}(k),
\end{aligned}
$$

where

$$
A_{\zeta}=\sum_{\lambda=1}^{\infty} \frac{Z_{\zeta}^{(\lambda)}\left(e, \tilde{k}_{F}\right)}{\epsilon^{\lambda}} \text { with } \zeta=0,1,2,3_{c}, 3_{s}, 4_{c}, 4_{s} .
$$

We have taken into account the exchange symmetry: $\Psi_{1, j} \leftrightarrow \Psi_{2, j}, a_{s} \rightarrow-a_{s}$, which was assumed in Ref. [62], and here it means that both $\Psi_{1, j}$ and $\Psi_{2, j}$ have the same wave function renormalization $Z_{\Psi}^{1 / 2}$.

Adding the counterterms to the original action, we obtain the renormalized action:

$$
\begin{aligned}
S_{\mathrm{ren}}= & \sum_{\alpha, j} \int d k^{B} \bar{\Psi}_{\alpha, j}^{B}\left(k^{B}\right) i\left(\gamma_{0} k_{0}^{B}+\tilde{\boldsymbol{\Gamma}} \cdot \tilde{\mathbf{K}}^{B}+\gamma_{d-m} \delta_{k}\right) \Psi_{\alpha, j}^{B}\left(k^{B}\right) \exp \left(\frac{\mathbf{L}_{\left(k^{B}\right)}^{2}}{\mu \tilde{k}_{F}^{B}}\right)+\frac{1}{2} \int d k^{B} \mathbf{L}_{\left(k^{B}\right)}^{2} a_{c}^{B^{\dagger}}\left(k^{B}\right) a_{c}^{B}\left(k^{B}\right) \\
& +\frac{1}{2} \int d k^{B} \mathbf{L}_{\left(k^{B}\right)}^{2} a_{s}^{B^{\dagger}}\left(k^{B}\right) a_{s}^{B}\left(k^{B}\right)-\frac{e_{c}^{B}}{\sqrt{N}} \sum_{\alpha, j} \int d k^{B} d q^{B} a_{c}^{B}\left(q^{B}\right) \bar{\Psi}_{\alpha, j}^{B}\left(k^{B}+q^{B}\right) \gamma_{0} \Psi_{\alpha, j}^{B}\left(k^{B}\right) \\
& +\frac{e_{s}^{B}}{\sqrt{N}} \sum_{\alpha, j}(-1)^{\alpha} \int d k^{B} d q^{B} a_{s}^{B}\left(q^{B}\right) \bar{\Psi}_{\alpha, j}^{B}\left(k^{B}+q^{B}\right) \gamma_{0} \Psi_{\alpha, j}^{B}\left(k^{B}\right),
\end{aligned}
$$

remembering that $\delta_{k^{B}}=\delta_{k}$. Here

$$
\begin{aligned}
k_{0}^{B} & =\frac{Z_{0}}{Z_{2}} k_{0}, \quad \tilde{\mathbf{K}}^{B}=\frac{Z_{1}}{Z_{2}} \tilde{\mathbf{K}}, \quad k_{d-m}^{B}=k_{d-m}, \quad \mathbf{L}_{\left(k^{B}\right)}=\mathbf{L}_{(k)}, \quad k_{F}^{B}=k_{F}=\mu \tilde{k}_{F}, \quad \Psi_{j}^{B}\left(k^{B}\right)=Z_{\Psi}^{\frac{1}{2}} \Psi_{j}(k), \\
a_{c}^{B}\left(k^{B}\right) & =Z_{a_{c}}^{\frac{1}{2}} a_{c}(k), \quad a_{s}^{B}\left(k^{B}\right)=Z_{a_{s}}^{\frac{1}{2}} a_{s}(k), \quad Z_{\Psi}=\frac{Z_{2}^{d-m+1}}{Z_{0} Z_{1}^{d-m-1}}, \quad Z_{a_{c}}=\frac{Z_{3_{s}} Z_{2}^{d-m}}{Z_{0} Z_{1}^{d-m-1}}, \quad Z_{a_{s}}=\frac{Z_{3_{c}} Z_{2}^{d-m}}{Z_{0} Z_{1}^{d-m-1}}, \\
e_{c}^{B} & =Z_{e_{c}} e_{c} \mu^{\frac{x}{2}}, \quad Z_{e_{c}}=\frac{Z_{4} Z_{2}^{\frac{d-m}{2}-1}}{\sqrt{Z_{0} Z_{3_{c}}} Z_{1}^{\frac{d-m-1}{2}}}, \quad e_{s}^{B}=Z_{e_{s}} e_{s} \mu^{\frac{x}{2}}, \quad Z_{e_{s}}=\frac{Z_{4} Z_{2}^{\frac{d-m}{2}-1}}{\sqrt{Z_{0} Z_{3_{s}}} Z_{1}^{\frac{d-m-1}{2}}},
\end{aligned}
$$

and

$$
Z_{\zeta}=1+A_{\zeta}
$$

As before, the superscript " $\mathrm{B}$ " denotes the bare fields, couplings, and momenta.

As before, we will use the same notations, namely, $z$ for the dynamical critical exponent, $\tilde{z}$ for the critical exponent along the extra spatial dimensions, $\beta_{k_{F}}$ for the beta function for $\tilde{k}_{F}$, and $\eta_{\psi}$ for the anomalous dimension of the fermions. Since we have two gauge fields now, we will use the symbols $\beta_{e_{c}}$ and $\beta_{e_{s}}$ to denote the beta functions for the couplings $e_{c}$ and $e_{s}$, respectively, which are explicitly given by

$$
\beta_{e_{c}}=\frac{\partial e_{c}}{\partial \ln \mu}, \quad \beta_{e_{s}}=\frac{\partial e_{s}}{\partial \ln \mu} .
$$


The anomalous dimensions of these two bosons are indicated by

$$
\eta_{a_{c}}=\frac{1}{2} \frac{\partial \ln Z_{a_{c}}}{\partial \ln \mu}, \quad \eta_{a_{s}}=\frac{1}{2} \frac{\partial \ln Z_{a_{s}}}{\partial \ln \mu} .
$$

\section{B. RG flows at one-loop order}

To one-loop order, the counterterms are given by $Z_{\zeta}=1+$ $\frac{Z_{\zeta}^{(1)}}{\epsilon}$. Here only

$$
Z_{0}^{(1)}=-\frac{u_{0}\left(\tilde{e}_{c}+\tilde{e}_{s}\right)}{N} \text { and } Z_{1}^{(1)}=-\frac{u_{1}\left(\tilde{e}_{c}+\tilde{e}_{s}\right)}{N}
$$

are nonzero, where

$$
\tilde{e}_{c}=\frac{e_{c}^{\frac{2(m+1)}{3}}}{\tilde{k}_{F}^{\frac{(m-1)(2-m)}{6}}} \text { and } \tilde{e}_{s}=\frac{e_{s}^{\frac{2(m+1)}{3}}}{\tilde{k}_{F}^{\frac{(m-1)(2-m)}{6}}} .
$$

The one-loop beta functions are now given by

$$
\begin{aligned}
& \beta_{k_{F}}=-\tilde{k}_{F}, \\
& (1-z) Z_{0}=-\beta_{e_{c}} \frac{\partial Z_{0}}{\partial e_{c}}-\beta_{e_{s}} \frac{\partial Z_{0}}{\partial e_{s}}+\tilde{k}_{F} \frac{\partial Z_{0}}{\partial \tilde{k}_{F}}, \\
& (1-\tilde{z}) Z_{1}=-\beta_{e_{c}} \frac{\partial Z_{1}}{\partial e_{c}}-\beta_{e_{s}} \frac{\partial Z_{1}}{\partial e_{s}}+\tilde{k}_{F} \frac{\partial Z_{1}}{\partial \tilde{k}_{F}}, \\
& \beta_{e_{c}}=-\frac{\epsilon+\frac{2-m}{m+1}(1-\tilde{z})+1-z}{2} e_{c}, \\
& \beta_{e_{s}}=-\frac{\epsilon+\frac{2-m}{m+1}(1-\tilde{z})+1-z}{2} e_{s}, \\
& \eta_{\psi}=\eta_{a_{c}}=\eta_{a_{s}}=\frac{1-z+(1-\tilde{z})(d-m-1)}{2} .
\end{aligned}
$$

Solving these equations, we get

$$
\begin{aligned}
-\frac{\beta_{e_{c}}}{e_{c}}= & -\frac{\beta_{e_{s}}}{e_{s}} \\
= & \frac{\epsilon}{2}+\frac{(m-1)(2-m)}{4(m+1)} \\
& -\frac{\left[(m+1) u_{0}+(2-m) u_{1}\right]\left(\tilde{e}_{c}+\tilde{e}_{s}\right)}{6 N} .
\end{aligned}
$$

Again, it is clear that for generic $m$, the order-by-order loop corrections are controlled not by $e_{c}$ and $e_{s}$, but by the effective couplings $\tilde{e}_{c}$ and $\tilde{e}_{s}$. Hence we need to compute the RG flows from the beta functions of these effective couplings, which are given by

$$
\begin{aligned}
-\frac{\beta_{\tilde{e}_{c}}}{\tilde{e}_{c}}= & -\frac{\beta_{\tilde{e}_{s}}}{\tilde{e}_{s}} \\
= & \frac{(m+1) \epsilon}{3} \\
& -\frac{(m+1)\left[(m+1) u_{0}+(2-m) u_{1}\right]\left(\tilde{e}_{c}+\tilde{e}_{s}\right)}{9 N} .
\end{aligned}
$$

The interacting fixed points are determined from the zeros of the above beta functions and take the form

$$
\tilde{e}_{c}^{*}+\tilde{e}_{s}^{*}=\frac{3 N \epsilon}{(m+1) u_{0}+(2-m) u_{1}}+O\left(\epsilon^{2}\right),
$$

which actually give rise to a fixed line, as found in Ref. [62] for the case of $m=1$. It can be checked that this is IR stable by computing the first derivative of the beta functions. Hence, we have proven that the fixed line feature survives for critical Fermi surfaces of dimensions more than one. The critical exponents at this stable fixed line take the same forms as in Eq. (24).

\section{Higher-loop corrections}

Using the same arguments as the single gauge field case, we will have the following nonzero $Z_{\zeta}^{(1)}$ 's:

$$
\begin{aligned}
& Z_{0}^{(1)}=-\frac{u_{0}\left(\tilde{e}_{c}+\tilde{e}_{s}\right)}{N \epsilon}-\frac{v_{0}\left(\tilde{e}_{s}+\tilde{e}_{c}\right)^{2}}{N^{2} \epsilon}, \\
& Z_{1}^{(1)}=-\frac{u_{1}\left(\tilde{e}_{s}+\tilde{e}_{c}\right)}{N \epsilon}-\frac{v_{1}\left(\tilde{e}_{c}+\tilde{e}_{s}\right)^{2}}{N^{2} \epsilon}, \\
& Z_{2}^{(1)}=-\frac{w\left(\tilde{e}_{c}+\tilde{e}_{s}\right)^{2}}{N^{2} \epsilon}, \\
& Z_{4_{s}}^{(1)}=-\frac{y_{s}\left(\tilde{e}_{c}+\tilde{e}_{s}\right)^{2}}{N^{2} \epsilon}, \quad Z_{4_{c}}^{(1)}=-\frac{y_{c}\left(\tilde{e}_{c}+\tilde{e}_{s}\right)^{2}}{N^{2} \epsilon},
\end{aligned}
$$

including the one- and two-loop corrections for $m=1$. This leads to the beta functions

$$
\frac{\beta_{\tilde{e}_{c}}}{\tilde{e}_{c}}=\frac{\beta_{\tilde{e}_{s}}}{\tilde{e}_{s}}=-\frac{2\left[2 u_{1}\left(\tilde{e}_{c}+\tilde{e}_{s}\right)+3 N\right] \epsilon}{9 N}+\frac{2\left[2\left(\tilde{e}_{c}+\tilde{e}_{s}\right)\left(2 u_{0} u_{1}+u_{1}^{2}+6 v_{0}+3 v_{1}-9 w\right)+3 N\left(2 u_{0}+u_{1}\right)\right]\left(\tilde{e}_{c}+\tilde{e}_{s}\right)}{27 N^{2}},
$$

which again have a continuous line of fixed points defined by

$$
\frac{\tilde{e}_{s}^{*}+\tilde{e}_{c}^{*}}{N}=\frac{3 \epsilon}{2 u_{0}+u_{1}}-\frac{18\left(2 v_{0}+v_{1}-3 w\right)}{\left(2 u_{0}+u_{1}\right)^{3}} \epsilon^{2}+O\left(\epsilon^{3}\right) .
$$

However, at three-loop order, there are diagrams giving rise to terms in the beta functions which are not proportional to $\left(\tilde{e}_{s}+\tilde{e}_{c}\right)$ raised to some integer power. Some of them are shown in Fig. 8, which are the Aslamazov-Larkin diagrams in the particle-particle channels contributing to the self-energy of the $a_{c}$ transverse gauge field. All 16 diagrams give a total contribution proportional to $4 e_{c}^{2}\left(\tilde{e}_{c}^{2}+\tilde{e}_{s}^{2}\right)$. Similarly, the Aslamazov-Larkin diagrams in the particle-hole channels will contribute with a term proportional to $4 e_{c}^{2}\left(\tilde{e}_{c}^{2}+\tilde{e}_{s}^{2}\right)$. One can also easily conclude that such corrections to the selfenergy of the $a_{s}$ transverse gauge field will be proportional to $4 e_{s}^{2}\left(\tilde{e}_{c}^{2}+\tilde{e}_{s}^{2}\right)$. Note that if the two fermions had carried the same charge under the two gauge gauge fields, then the contributions would have been proportional to $4 e_{c}^{2}\left(\tilde{e}_{c}+\tilde{e}_{s}\right)^{2}$ and $4 e_{s}^{2}\left(\tilde{e}_{c}+\tilde{e}_{s}\right)^{2}$, respectively, for the $a_{c}$ and $a_{s}$ fields, leading to the preservation of the fixed line feature.

For $m>1$, the UV/IR mixing will render the higher-loop corrections to be $k_{F}$-suppressed and hence they will have no 


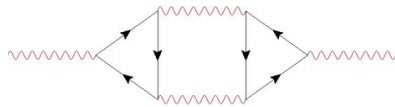

(a)

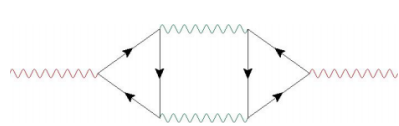

(e)

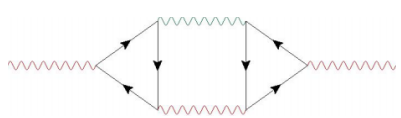

(i)

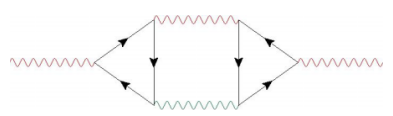

(m)

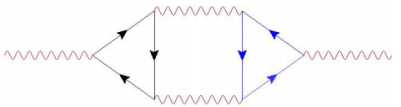

(b)

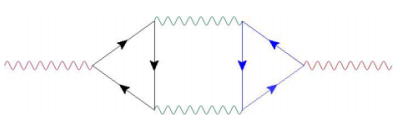

(f)

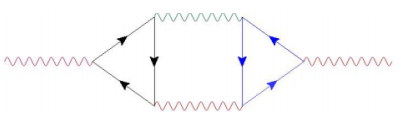

(j)

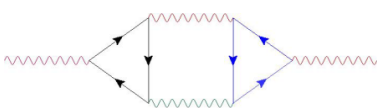

(n)

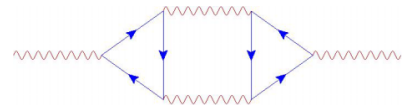

(c)

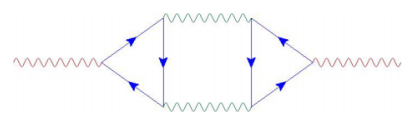

(g)

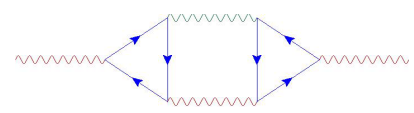

(k)

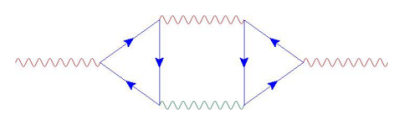

(o)

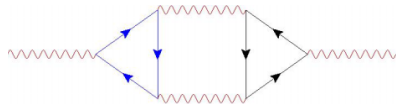

(d)

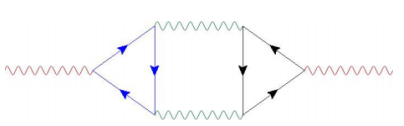

(h)

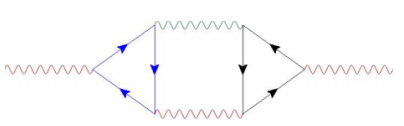

(1)

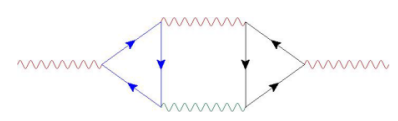

(p)

FIG. 8. Contributions from the Aslamazov-Larkin diagrams in the particle-particle channels to the three-loop boson self-energy of the $a_{c}$ transverse gauge field. The red and green wavy lines denote the $a_{c}$ and $a_{s}$ propagators, respectively. The black and blue solid lines with arrows represent the $\psi_{1, \pm, j}$ and $\psi_{2, \pm, j}$ fermion propagators, respectively. All 16 diagrams give a total contribution proportional to $4 e_{c}^{2}\left(\tilde{e}_{c}^{2}+\tilde{e}_{s}^{2}\right)$.

effect on the fixed line. Therefore, the fixed line feature is generically not altered by going to higher loops.

\section{CONCLUSION}

In this paper, we have applied the dimensional regularization scheme, developed for non-Fermi liquids arising at Ising-nematic quantum critical point, to the case of non-Fermi liquids arising from transverse gauge field couplings with finite-density fermions. This has allowed us to access the interacting fixed points perturbatively in an expansion in $\epsilon$, which is the difference between the upper critical dimension $\left(d_{c}=m+\frac{3}{m+1}\right)$ and the actual physical dimension $\left(d_{\mathrm{phys}}=\right.$ $m+1)$ of the theory, for a Fermi surface of dimension $m$. We have extracted the scaling behavior for the case of one and two $U(1)$ gauge fields.

There is a crucial difference in the matrix structure of the couplings in the cases of Ising-nematic order parameter and gauge fields. This arises from the fact that the fermions on the antipodal points of the Fermi surface couple to the Isingnematic order parameter (transverse gauge) with the same (opposite) sign(s). Hence, although we get the same values of critical dimension and critical exponents, the differences will show up in the renormalization of some physical quantities like the $2 k_{F}$ scattering (backscattering involving an operator that carries a momentum $2 k_{F}$ ) amplitudes. This operator can also be identified as the CDW instability. In particular, we have found that this CDW ordering is enhanced in the vicinity of the non-Fermi liquid critical point for transverse gauge field(s) for $m=1$, in contrast to the Ising-nematic scenario.

The $U(1) \times U(1)$ is particularly interesting in the context of recent works which show that this scenario is useful to describe the phenomena of deconfined Mott transition and deconfined metal-metal transition [62]. In Ref. [62] Zou and Chowdhury found that in $(2+1)$ spacetime dimensions and at one-loop order, these systems exhibited a continuous line of stable fixed points, rather than a single one. Their method involved modifying the bosonic dispersion (such that it becomes nonanalytic in the momentum space) and then carrying out a double expansion in two small parameters $[9,23]$. Our method avoids this issue by employing the dimensional regularization scheme. We also have the advantage that we could analyze a critical Fermi surface of generic dimensions and also perform higher-loop diagrams giving order by order corrections in $\epsilon$. The discovery of a fixed line for the $U(1) \times U(1)$ theory in Ref. [62] raised the question whether this feature survives when we consider either higher dimensions or higher loops. Our computations show that definitely higher dimensions do not reduce the fixed line to discrete fixed points (or no fixed point at all). Regarding higher-loop corrections, we have not performed those explicitly, but through arguments based on the previous results for the Ising-nematic critical points $[28,30,31]$, we have predicted that although the two-loop corrections will not affect the fixed line, the presence of certain three-loop diagrams will. If the fixed line feature is destroyed by three-loop and/or higher-order corrections for $m=1$, this will have the following possibilities for Ref. [62]: (1) The fixed line degenerates into fixed points, which might be stable or unstable. (2) The zeros of the beta functions have no finite solution (i.e. no fixed point exists). The physical consequences of these scenarios have been explained in detail in Sec. V of Ref. [62].

In the future, it will be worthwhile to carry out this entire procedure for the case of $S U$ (2) gauge fields [62]. It is also possible that the non-Fermi liquid fixed point or line for $m=1$ is masked by a CDW phase. Extending our RG analysis to a situation where fluctuations due to the transverse gauge field(s) and CDW are treated on equal footing would be an interesting problem for future study. Another direction is to compute the RG flows for superconducting instabilities in the presence of the transverse gauge field(s), as was done in Ref. [33] for the Ising-nematic order parameter. 


\section{ACKNOWLEDGMENTS}

We thank Debanjan Chowdhury for stimulating discussions. We are especially grateful to Andres Schlief for valuable comments on the manuscript. We also than Sung-Sik Lee for pointing out that Aslamasov-Larkin diagrams will affect the fixed line feature at three-loop order.

\section{APPENDIX: COMPUTATION OF THE FEYNMAN DIAGRAMS AT ONE-LOOP ORDER}

\section{One-loop boson self-energy}

In this subsection, we compute the one-loop boson self-energy:

$$
\Pi_{1}(q)=-e^{2} \mu^{x} \int d k \operatorname{Tr}\left[\gamma_{0} G_{0}(k+q) \gamma_{0} G_{0}(k)\right]=2 e^{2} \mu^{x} \int d k \frac{k_{0}\left(k_{0}+q_{0}\right)-\tilde{\mathbf{K}} \cdot(\tilde{\mathbf{K}}+\tilde{\mathbf{Q}})-\delta_{q} \delta_{k+q}}{\left[\mathbf{K}^{2}+\delta_{k}^{2}\right]\left[(\mathbf{K}+\mathbf{Q})^{2}+\delta_{k+q}^{2}\right]} e^{-\frac{\mathbf{L}_{(k)+\mathbf{L}_{(k+q)}^{2}}}{\mu \hat{k}_{F}}} .
$$

We first integrate over $k_{d-m}$ to obtain [68]

$$
\begin{aligned}
\Pi_{1}(q)= & e^{2} \mu^{x} \int \frac{d \mathbf{L}_{(k)} d \mathbf{K}}{(2 \pi)^{d}} \frac{2 k_{0}\left(k_{0}+q_{0}\right)-\mathbf{K} \cdot(\mathbf{K}+\mathbf{Q})}{|\mathbf{K}||\mathbf{K}+\mathbf{Q}|\left[\left(\delta_{q}+2 \mathbf{L}_{(q)}^{i} \mathbf{L}_{(k)}^{i}\right)^{2}+(|\mathbf{K}+\mathbf{Q}|+|\mathbf{K}|)^{2}\right]}(|\mathbf{K}+\mathbf{Q}|+|\mathbf{K}|) e^{-\frac{\mathbf{L}_{(k)}^{2}+\mathbf{L}_{(k+q)}^{2}}{\mu \hat{k}_{F}}} \\
& -e^{2} \mu^{x} \int \frac{d \mathbf{L}_{(k)} d \mathbf{K}}{(2 \pi)^{d}} \frac{|\mathbf{K}+\mathbf{Q}|+|\mathbf{K}|}{\left(\delta_{q}+2 \mathbf{L}_{(q)}^{i} \mathbf{L}_{(k)}^{i}\right)^{2}+(|\mathbf{K}+\mathbf{Q}|+|\mathbf{K}|)^{2}} \times e^{-\frac{\mathbf{L}_{(k)}^{2}+\mathbf{L}_{(k+q)}^{2}}{\mu \tilde{k}_{F}}},
\end{aligned}
$$

where we have chosen the coordinate system such that $\mathbf{L}_{(q)}=\left(q_{d-m+1}, 0,0, \ldots, 0\right)$. Since the problem is rotationally invariant in these directions and $\Pi_{1}(q)$ depends only on the magnitude of $\mathbf{L}_{(q)}$, the final result is independent of this choice.

Making a change of variable, $u=\delta_{q}+2 q_{d-m+1} k_{d-m+1}$, and integrating over $u$, we get

$$
I \equiv \int \frac{d k_{d-m+1}}{2 \pi} \frac{1}{\left(\delta_{q}+2 q_{d-m+1} k_{d-m+1}\right)^{2}+(|\mathbf{K}+\mathbf{Q}|+|\mathbf{K}|)^{2}}=\frac{1}{4\left|\mathbf{L}_{(q)}\right|(|\mathbf{K}+\mathbf{Q}|+|\mathbf{K}|)} .
$$

The rest of $\mathbf{L}_{(k)}$ integrals evaluate to $J^{m-1}$, where

$$
J \equiv \int_{-\infty}^{\infty} \frac{d y}{2 \pi} \exp \left(\frac{-2 y^{2}}{\mu \tilde{k}_{F}}\right)=\sqrt{\frac{\mu \tilde{k}_{F}}{8 \pi}} .
$$

Hence the self-energy expression reduces to

$$
\Pi_{1}(q)=\frac{e^{2} \mu^{x}}{2^{m+1}\left|\mathbf{L}_{(q)}\right|}\left(\frac{\mu \tilde{k}_{F}}{2 \pi}\right)^{\frac{m-1}{2}} I_{1}(d-m, \mathbf{Q}),
$$

where

$$
I_{1}(d-m, \mathbf{Q})=\int \frac{d \mathbf{K}}{(2 \pi)^{d-m}}\left[\frac{k_{0}\left(k_{0}+q_{0}\right)-\tilde{\mathbf{K}} \cdot(\tilde{\mathbf{K}}+\tilde{\mathbf{Q}})}{|\mathbf{K}+\mathbf{Q}||\mathbf{K}|}-1\right] .
$$

The $(d-m)$-dimensional integral in $I_{1}(d-m, \mathbf{Q})$ can be done using the Feynman parametrization formula

$$
\frac{1}{A^{\alpha} B^{\beta}}=\frac{\Gamma(\alpha+\beta)}{\Gamma(\alpha) \Gamma(\beta)} \int_{0}^{1} d t \frac{t^{\alpha-1}(1-t)^{\beta-1}}{[t A+(1-t) B]^{\alpha+\beta}} .
$$

Substituting $\alpha=\beta=1 / 2, A=|\mathbf{K}+\mathbf{Q}|^{2}$ and $B=|\mathbf{K}|^{2}$, we get

$$
I_{1}(d-m, \mathbf{Q})=\frac{1}{\pi(2 \pi)^{d-m}} \int_{0}^{1} \frac{d t}{\sqrt{t(1-t)}} \int d \mathbf{K}\left[\frac{k_{0}\left(k_{0}+q_{0}\right)-\tilde{\mathbf{K}} \cdot(\tilde{\mathbf{K}}+\tilde{\mathbf{Q}})}{x|\mathbf{K}+\mathbf{Q}|^{2}+(1-t) \mathbf{K}^{2}}-1\right] .
$$

Introducing the new variable $\mathbf{u}=\mathbf{K}+t \mathbf{Q}, I_{1}$ reduces to

$$
I_{1}(d-m, \mathbf{Q})=\frac{1}{\pi(2 \pi)^{d-m}} \int_{0}^{1} \frac{d t}{\sqrt{t(1-t)}} \int d^{d-m} \mathbf{u}\left\{\frac{2\left[u_{0}^{2}-t(1-t) q_{0}^{2}\right]-2 \mathbf{u}^{2}}{\mathbf{u}^{2}+t(1-t) \mathbf{Q}^{2}}\right\} .
$$

Again, we use another new variable $\mathbf{v}$, defined by $\mathbf{u}=\sqrt{t(1-t)} \mathbf{v}$, so that

$$
I_{1}(d-m, \mathbf{Q})=-\frac{2^{-2 d+2 m+1} \pi^{-d+m+\frac{1}{2}} \Gamma\left(\frac{d-m+1}{2}\right)}{\Gamma\left(\frac{d-m+2}{2}\right)} \int d^{d-m} \mathbf{v} \frac{q_{0}^{2}+\tilde{\mathbf{v}}^{2}}{\mathbf{v}^{2}+\mathbf{Q}^{2}} .
$$


Using

$$
\int_{0}^{\infty} d y \frac{y^{n_{1}}}{\left(y^{2}+C\right)^{n_{2}}}=\frac{\Gamma\left(\frac{n_{1}+1}{2}\right) \Gamma\left(n_{2}-\frac{n_{1}+1}{2}\right)}{2 \Gamma\left(n_{2}\right)} C^{\frac{n_{1}+1}{2}-n_{2}}
$$

and the volume of the ( $n-1)$-sphere (at the boundary of the $n$-ball of unit radius)

$$
S^{n-1} \equiv \int d \Omega_{n}=\frac{2 \pi^{n / 2}}{\Gamma(n / 2)}
$$

we finally obtain the one-loop boson self-energy to be

$$
\Pi_{1}(k)=-\frac{\beta(d, m) e^{2} \mu^{x}}{\left|\mathbf{L}_{(q)}\right|}\left(\mu \tilde{k}_{F}\right)^{\frac{m-1}{2}}\left[k_{0}^{2}+(m+1-d) \tilde{\mathbf{K}}^{2}\right]|\mathbf{K}|^{d-m-2},
$$

with

$$
\beta(d, m)=\frac{1}{2^{m+1}}\left(\frac{1}{2 \pi}\right)^{\frac{m-1}{2}} \frac{2^{-2 d+2 m+1} \pi^{\frac{-d+m+3}{2}} \Gamma(d-m) \Gamma(m+1-d)}{\Gamma^{2}\left(\frac{d-m+2}{2}\right) \Gamma\left(\frac{m+1-d}{2}\right)}=\frac{2^{\frac{1+m-4 d}{2}} \pi^{\frac{4-d}{2}} \Gamma(d-m) \Gamma(m+1-d)}{\Gamma^{2}\left(\frac{d-m+2}{2}\right) \Gamma\left(\frac{m+1-d}{2}\right)} .
$$

\section{One-loop fermion self-energy}

Here we compute the one-loop fermion self-energy $\Sigma_{1}(q)$ by using the dressed propagator for boson which includes the one-loop self-energy $\Pi_{1}(k)$ :

$$
\Sigma_{1}(q)=\frac{e^{2} \mu^{x}}{N} \int d k \gamma_{0} G_{0}(k+q) \gamma_{0} D_{1}(k)=\frac{i e^{2} \mu^{x}}{N} \int d k D_{1}(k) \frac{\tilde{\boldsymbol{\Gamma}} \cdot(\tilde{\mathbf{K}}+\tilde{\mathbf{Q}})+\gamma_{d-m} \delta_{k+q}-\gamma_{0}\left(k_{0}+q_{0}\right)}{(\mathbf{K}+\mathbf{Q})^{2}+\delta_{k+q}^{2}} e^{-\frac{\mathbf{L}_{(k+q)}^{2}}{\mu \hat{k}_{F}}} .
$$

Integrating over $k_{d-m}$, we get

$$
\Sigma_{1}(q)=\frac{i e^{2} \mu^{x}}{2 N} \int \frac{d^{d} k}{(2 \pi)^{d}} D_{1}(k) \frac{\tilde{\boldsymbol{\Gamma}} \cdot(\tilde{\mathbf{K}}+\tilde{\mathbf{Q}})-\gamma_{0}\left(k_{0}+q_{0}\right)}{|\mathbf{K}+\mathbf{Q}|} \times e^{-\frac{\mathbf{L}_{(k+q)}^{2}}{\mu \vec{k}_{F}}} .
$$

Since only $D_{1}(k) e^{-\frac{\mathbf{L}_{(k+q)}^{2}}{k_{F}}}$ depends on $\mathbf{L}_{(k)}$, let us first perform the integral:

$$
\begin{aligned}
I_{2}(k) & \equiv \int \frac{d \mathbf{L}_{(k)}}{(2 \pi)^{m}} \frac{e^{-\frac{\mathbf{L}_{(q+k)}^{2}}{k_{F}}} \frac{\mathbf{L}_{(k)}^{2}+\beta(d, m) e^{2} \mu^{x}\left(\mu \tilde{k}_{F}\right)^{\frac{m-1}{2}} \frac{|\mathbf{K}|^{d-m}}{\left|\mathbf{L}_{(k)}\right|} \times\left[d-m-1+(m-d) \frac{k_{0}^{2}}{|\mathbf{K}|^{2}}\right]}{\pi^{\frac{2-m}{2}}}}{3 \times 2^{m-1} \Gamma(m / 2)|\sin [(m+1) \pi / 3]|\left\{\beta(d, m) e^{2} \mu^{x}\left(\mu \tilde{k}_{F}\right)^{\frac{m-1}{2}}|\mathbf{K}|^{d-m-2} \times\left[k_{0}^{2}+(m+1-d) \tilde{\mathbf{K}}^{2}\right]\right\}^{\frac{2-m}{3}}} .
\end{aligned}
$$

Now the expression for the self-energy can be written as

$$
\Sigma_{1}(q)=\frac{i e^{2(m+1) / 3} \mu^{x(m+1) / 3} \pi^{\frac{2-m}{2}} \times I_{3}(d-m, \mathbf{Q})}{6 N \times 2^{m-1} \Gamma(m / 2)\left|\sin \left(\frac{m+1}{3} \pi\right)\right|[\beta(d, m)]^{\frac{2-m}{3}}\left(\mu \tilde{k}_{F}\right)^{(m-1)(2-m) / 6}},
$$

where

$$
\begin{aligned}
I_{3}(d-m, \mathbf{Q})= & \int \frac{d \mathbf{K}}{(2 \pi)^{d-m}} \frac{\tilde{\boldsymbol{\Gamma}} \cdot(\tilde{\mathbf{K}}+\tilde{\mathbf{Q}})-\gamma_{0}\left(k_{0}+q_{0}\right)}{\left\{\left[k_{0}^{2}+(m+1-d) \tilde{\mathbf{K}}^{2}\right]|\mathbf{K}|^{d-m-2}\right\}^{\frac{2-m}{3}}|\mathbf{K}+\mathbf{Q}|} \\
= & \frac{\Gamma\left[\frac{2 d+m^{2}+3-m(d+2)}{6}\right]}{\Gamma\left(\frac{1}{2}\right) \Gamma\left(\frac{2-m}{3}\right) \Gamma\left[\frac{(d-m-2)(2-m)}{6}\right]} \int_{0}^{1} d t_{1} \int_{0}^{1-t_{1}} d t_{2} \frac{\left(1-t_{1}-t_{2}\right)^{\frac{(d-m-2)(2-m)}{6}-1}}{t_{1}^{\frac{m+1}{3}} \sqrt{t_{2}}} \\
& \times \int \frac{d \mathbf{K}}{(2 \pi)^{d-m}} \frac{\tilde{\boldsymbol{\Gamma}} \cdot(\tilde{\mathbf{K}}+\tilde{\mathbf{Q}})-\gamma_{0}\left(1-t_{2}\right) q_{0}}{\left[t_{1}(m+1-d) \tilde{\mathbf{K}}^{2}+t_{2}(\tilde{\mathbf{K}}+\tilde{\mathbf{Q}})^{2}+\left(1-t_{1}-t_{2}\right) \tilde{\mathbf{K}}^{2}+q_{0}^{2} t_{2}\left(1-t_{2}\right)+k_{0}^{2}\right]^{\frac{2 d+m^{2}+3-m(d+2)}{6}}} \\
= & \frac{\pi^{\frac{m-d-1}{2}} \Gamma\left[\frac{3-(d-m)(m+1)}{6}\right]}{2^{d-m} \Gamma\left(\frac{2-m}{3}\right) \Gamma\left[\frac{m^{2}-d(m-2)-4}{6}\right]} \int_{0}^{1} d t_{1} \int_{0}^{1-t_{1}} d t_{2}\left\{\frac{\left(1-t_{1}-t_{2}\right)^{\frac{(d-m-2)(2-m)}{6}-1} \gamma_{0}\left(1-t_{2}\right) q_{0}-(\tilde{\boldsymbol{\Gamma}} \cdot \tilde{\mathbf{Q}})\left[\frac{\left(1+m t_{1}-d t_{1}-t_{2}\right)}{1+m t_{1}-d t_{1}}\right]}{t_{1}^{\frac{m+1}{3}}} \sqrt{t_{2}}\right.
\end{aligned}
$$




$$
\left.\times\left[\frac{\tilde{\mathbf{Q}}^{2} t_{2}\left(1+m t_{1}-d t_{1}-t_{2}\right)}{\left(1+m t_{1}-d t_{1}\right)^{2}}+\frac{q_{0}^{2} t_{2}\left(1-t_{2}\right)}{1+m t_{1}-d t_{1}}\right]^{\frac{(d-m)(m+1)-3}{6}}\left[\Theta\left(1+m t_{1}-d t_{1}\right)+f a c \times \Theta\left(d t_{1}-1-m t_{1}\right)\right]\right\} .
$$

where $f a c=(-1)^{\frac{(m-2)(d-m)}{3}} i^{d-m+1}\left\{(-1)^{\frac{1}{6}(m-2)(m-d)} \cos \left[\frac{(m+1)(d-m)}{6} \pi\right]-\cos \left(\frac{d-m}{2} \pi\right)\right\} \csc \left[\frac{(m-2)(d-m)}{6} \pi\right]$. From this expression, it is clear that $I_{3}(d-m, \mathbf{Q})$ blows up when the argument of the gamma function in the numerator blows up: $\frac{3-(d-m)(m+1)}{6}=0$. This implies that $\Sigma_{1}(q)$ blows up logarithmically in $\Lambda$ at the critical dimension

$$
d_{c}(m)=m+\frac{3}{m+1} .
$$

The integrals over $t_{1}$ and $t_{2}$ are convergent, but their values have to be computed numerically for a given $m$.

Expanding in $\epsilon$ defined as $d=m+\frac{3}{m+1}-\epsilon$, we obtain

$$
\Sigma_{1}(q)=-\frac{i e^{\frac{2(m+1)}{3}}\left[u_{0}\left(\frac{\mu}{\left|q_{0}\right|}\right)^{\frac{m+1}{3} \epsilon} \gamma_{0} q_{0}+u_{1}\left(\frac{\mu}{|\tilde{\mathbf{Q}}|}\right)^{\frac{m+1}{3} \epsilon}(\tilde{\boldsymbol{\Gamma}} \cdot \tilde{\mathbf{Q}})\right]}{N \tilde{k}^{\frac{(m-1)(2-m)}{6}} \epsilon}+\text { finite terms }
$$

where $u_{0}, u_{1} \geqslant 0$. Numerically, we find

$$
\left\{\begin{array}{ll}
u_{0}=0.0201044, \quad u_{1}=1.85988 & \text { for } m=1 \\
u_{0}=u_{1}=0.0229392 & \text { for } m=2
\end{array} .\right.
$$

\section{One-loop vertex correction}

In general, the one-loop fermion-boson vertex function $\Gamma_{1}(k, q)$ depends on both $k$ and $q$. In order to extract the leading $1 / \epsilon$ divergence, however, it is enough to look at the $q \rightarrow 0$ limit. In this limit, we get

$$
\begin{aligned}
\Gamma_{1}(k, 0) & =\frac{e^{2} \mu^{x}}{N} \int \frac{d^{d+1} q}{(2 \pi)^{d+1}} \gamma_{0} G_{0}(q) \gamma_{0} G_{0}(q) \gamma_{0} D_{1}(q-k) \\
& =\frac{e^{2} \mu^{x}}{N} \int \frac{d^{d+1} q}{(2 \pi)^{d+1}} D_{1}(q-k) \gamma_{0}\left[\frac{1}{\mathbf{Q}^{2}+q_{d-m}^{2}}-\frac{2 q_{0}^{2}}{\left(q_{0}^{2}+\tilde{\mathbf{Q}}^{2}+q_{d-m}^{2}\right)^{2}}\right] e^{-\frac{2 \mathbf{L}_{(q)}^{2}}{\mu \hat{k}_{F}}} \\
& =\frac{e^{2} \mu^{x}}{2 N} \int \frac{d \mathbf{Q} d \mathbf{L}_{(q)}}{(2 \pi)^{d}} D_{1}(q) \gamma_{0} \frac{(\tilde{\mathbf{Q}}+\tilde{\mathbf{K}})^{2}}{\left[\left(q_{0}+k_{0}^{2}\right)^{2}+(\tilde{\mathbf{Q}}+\tilde{\mathbf{K}})^{2}\right]^{3 / 2}} e^{-\frac{2 \mathbf{L}_{(q+k)}^{2}}{\mu \bar{k}_{F}}} .
\end{aligned}
$$

Using Eq. (A17), the above expression reduces to

$$
\Gamma_{1}(k, 0)=\frac{e^{2} \mu^{x} \gamma_{0}}{N} \frac{\pi^{\frac{m}{2}+1-d}}{3 \times 2^{d} \Gamma(m / 2)\left|\sin \left(\frac{m+1}{3} \pi\right)\right|\left[\beta(d, m) e^{2} \mu^{x}\left(\mu \tilde{k}_{F}\right)^{\frac{m-1}{2}}\right]^{\frac{2-m}{3}}} \times I_{4}(d, m),
$$

where

$$
\begin{aligned}
I_{4}(d, m)= & \int \frac{d \mathbf{Q}}{\left\{|\mathbf{Q}|^{d-m-2}\left[q_{0}^{2}+(m+1-d) \tilde{\mathbf{Q}}^{2}\right]\right\}^{\frac{2-m}{3}}} \frac{(\tilde{\mathbf{Q}}+\tilde{\mathbf{K}})^{2}}{\left[\left(q_{0}+k_{0}^{2}\right)^{2}+(\tilde{\mathbf{Q}}+\tilde{\mathbf{K}})^{2}\right]^{3 / 2}} \\
= & \int_{0}^{1} d t_{1} \int_{0}^{1-t_{1}} d t_{2} \frac{\Gamma\left[\frac{2 d+m^{2}+9-m(d+2)}{6}\right] t_{1}^{-\frac{m+1}{3}} t_{2}^{\frac{1}{2}}\left(1-t_{1}-t_{2}\right)^{\frac{(d-m-2)(2-m)}{6}-1}}{\Gamma\left(\frac{3}{2}\right) \Gamma\left(\frac{2-m}{3}\right) \Gamma\left[\frac{(d-m-2)(2-m)}{6}\right]} \\
& \times \int \frac{d \mathbf{Q}}{(2 \pi)^{d-m}} \frac{(\tilde{\mathbf{Q}}+\tilde{\mathbf{K}})^{2}}{\left[t_{1}(m+1-d) \tilde{\mathbf{Q}}^{2}+t_{2}(\tilde{\mathbf{Q}}+\tilde{\mathbf{K}})^{2}+\left(1-t_{1}-t_{2}\right) \tilde{\mathbf{Q}}^{2}+k_{0}^{2} t_{2}\left(1-t_{2}\right)+q_{0}^{2}\right]^{\frac{-(d+2) m+2 d+m^{2}+9}{6}}} \\
= & \int_{0}^{1} d t_{1}^{1-t_{1}} d t_{2} \frac{\Gamma\left[\frac{2 d+m^{2}+9-m(d+2)}{6}\right] t_{1}^{-\frac{m+1}{3}} t_{2}^{\frac{1}{2}}\left(1-t_{1}-t_{2}\right)^{\frac{(d-m-2)(2-m)}{6}-1}}{\Gamma\left(\frac{3}{2}\right) \Gamma\left(\frac{2-m}{3}\right) \Gamma\left[\frac{(d-m-2)(2-m)}{6}\right]} \\
& \times \frac{\Gamma\left[\frac{(d-m)(2-m)}{6}+1\right]}{\Gamma\left[\frac{m^{2}-(d+2) m+2 d+9}{6}\right]} \frac{J_{1}}{\left(1+m t_{1}-d t_{1}\right)^{\frac{(d-m)(m-2)-6}{6}}} .
\end{aligned}
$$


Here

$$
\begin{aligned}
J_{1} & =\int \frac{d \tilde{\mathbf{Q}}}{(2 \pi)^{d-m}} \frac{\tilde{\mathbf{Q}}^{2}+A^{2}}{\left(\tilde{\mathbf{Q}}^{2}+B\right)^{\frac{(d-m)(m-2)-6}{6}}} \\
= & \frac{B^{\frac{5 d+m^{2}+3-(d+5) m}{6}} \Gamma\left[\frac{(d-m)(m-5)-9}{6}\right]}{3 \times 2^{d-m+1} \pi^{\frac{d-m+1}{2}} \Gamma\left[\frac{(d-m)(m-2)}{6}-1\right]}\left\{[(d-m)(m-5)-9] A^{2}+3(d-m-1) B\right\},
\end{aligned}
$$

where $B \equiv \frac{\tilde{\mathbf{K}}^{2} t_{2}\left(1+m t_{1}-d t_{1}-t_{2}\right)}{\left(1+m t_{1}-d t_{1}\right)^{2}}+\frac{k_{0}^{2} t_{2}\left(1-t_{2}\right)}{1+m t_{1}-d t_{1}}$ and $A^{2}=\left(\frac{1+m t_{1}-d t_{1}-t_{2}}{1+m t_{1}-d t_{1}}\right)^{2} \tilde{\mathbf{K}}^{2}$.

As demonstrated below, we find that although the final value has to be calculated numerically, the expression for $\Gamma_{1}(k, 0)$ does not have any pole in $\epsilon=m+\frac{3}{m+1}-\epsilon$ and is therefore nondivergent:

$$
\Gamma_{1}(k, 0)=\left\{\begin{array}{ll}
-\frac{e^{2 / 3} \mu^{2 \epsilon / 3} \gamma_{0}}{N\left[\beta\left(\frac{5}{2}, 1\right)\right]^{\frac{1}{3}}} \times \frac{\Gamma\left(\frac{5}{4}\right)}{180 \sqrt{3} \pi^{9 / 4} \Gamma\left(-\frac{5}{4}\right) \Gamma\left(-\frac{1}{12}\right) \Gamma\left(\frac{1}{3}\right)^{2}} \int_{0}^{1} d t_{1} \int_{0}^{1-t_{1}} d t_{2} \frac{t_{2}^{\frac{1}{2}} B^{3 / 2}\left(B-10 A^{2}\right)}{t_{1}^{\frac{2}{3}}\left(1-t_{1}-t_{2}\right)^{\frac{13}{12}}} & \text { for } m=1 \\
0 & \text { for } m=2
\end{array} .\right.
$$

\section{Renormalization of the $2 k_{F}$ scattering amplitude}

The one-loop corrections to the $2 k_{F}$ scattering amplitude $g_{2 k_{F}}$, captured by Fig. 7 , can be obtained as

$$
\frac{i e^{2} \mu^{x} g_{2 k_{F}}}{N} \int d q \gamma_{0}^{T} G_{0}^{T}(q) \gamma_{0} G_{0}(-q) \gamma_{0} D_{1}(q-k)
$$

where the superscript $T$ denotes transpose of the corresponding matrix. If $d-m=2$, we have $\gamma_{0}^{T}=-\sigma_{y}=-\gamma_{0}, \gamma_{1}^{T}=\sigma_{z}=\gamma_{1}$, and $\gamma_{2}^{T}=\sigma_{x}=\gamma_{2}$. For $2<d<5 / 2$, we generalize this as

$$
\gamma_{0}^{T}=-\gamma_{0}, \quad \gamma_{\chi}^{T}=\gamma_{\chi}, \text { for } \chi=1, \ldots, d-m .
$$

Using this, we obtain the one-loop correction

$$
\begin{aligned}
& \delta g_{2 k_{F}}^{(1)}=\frac{i e^{2} \mu^{x} g_{2 k_{F}}}{N} \int d q \gamma_{0} \frac{\Gamma^{T} \cdot \mathbf{Q}+\gamma_{0}^{T} \delta_{q}}{\mathbf{Q}^{2}+\delta_{q}^{2}} \gamma_{0} \frac{-\boldsymbol{\Gamma} \cdot \mathbf{Q}+\gamma_{d-m} \delta_{-q}}{\mathbf{Q}^{2}+\delta_{-q}^{2}} \gamma_{d-m} D_{1}(q-k) \\
& =-\frac{i e^{2} \mu^{x} g_{2 k_{F}}}{N} \int d q \gamma_{0} \frac{q_{0}^{2}+\tilde{\mathbf{Q}}^{2}+\delta_{q} \delta_{-q}}{\left(\mathbf{Q}^{2}+\delta_{q}^{2}\right)\left(\mathbf{Q}^{2}+\delta_{-q}^{2}\right)} D_{1}(q-k)+\text { terms irrelevant for } 2 k_{F} \text { scattering } \\
& =-\frac{i e^{2} \mu^{x} g_{2 k_{F}}}{2 N} \int \frac{d \mathbf{Q} d \mathbf{L}_{(q)}}{(2 \pi)^{d}} \frac{\gamma_{0}|\mathbf{Q}+\mathbf{K}|}{(\mathbf{Q}+\mathbf{K})^{2}+\mathbf{L}_{(q+k)}^{4}} \frac{e^{-\frac{\mathbf{L}_{(q)}^{2}}{k_{F}}}}{\mathbf{L}_{(q)}^{2}-\beta(d, m) e^{2} \mu^{x}\left(\mu \tilde{k}_{F}\right)^{\frac{m-1}{2}} \frac{|\mathbf{Q}|^{d-m-2}}{\left|\mathbf{L}_{(q)}\right|}\left[(m+1-d)|\tilde{\mathbf{Q}}|^{2}+q_{0}^{2}\right]} .
\end{aligned}
$$

In order to extract the divergent part, we can set $\mathbf{L}_{(k)}=0$, and scale $\mathbf{L}_{(q)}$ as $\left[e^{2} \mu^{x}\left(\mu \tilde{k}_{F}\right)^{\frac{m-1}{2}}\right]^{1 / 3} \mathbf{L}_{(q)}\left[\right.$ in addition to setting $\mathbf{L}_{(q)}$ to zero everywhere except $D_{1}(q)$ ] for the leading order behavior in $\tilde{e}$. This finally gives the expression

$$
\begin{aligned}
\delta g_{2 k_{F}}^{(1)} & =-\frac{i e^{2} \mu^{x} g_{2 k_{F}}}{2 N} \int \frac{d \mathbf{Q} d \mathbf{L}_{(q)}}{(2 \pi)^{d}} \frac{\gamma_{0}}{|\mathbf{Q}+\mathbf{K}|} \frac{\left|\mathbf{L}_{(q)}\right|}{\left|\mathbf{L}_{(q)}\right|^{3}-\beta(d, m)|\mathbf{Q}|^{d-m-2}\left[(m+1-d)|\tilde{\mathbf{Q}}|^{2}+\left(q_{0}+k_{0}\right)^{2}\right]} \frac{1}{\left[e^{2} \mu^{x}\left(\mu \tilde{k}_{F}\right)^{\frac{m-1}{2}}\right]^{\frac{2-m}{3}}} \\
& = \begin{cases}\frac{i e^{\frac{4}{3}\left(\frac{\mu}{\left|k_{0}\right|}\right)^{\frac{2 \epsilon}{3}} g_{2 k_{F}}}}{N} \times \frac{-0.0774559 \gamma_{0}}{\epsilon} & \text { for } m=1, \\
0 & \text { for } m=2\end{cases}
\end{aligned}
$$

modulo terms irrelevant for the renormalization of $2 k_{F}$ scattering.

[1] T. Holstein, R. E. Norton, and P. Pincus, de Haas-van Alphen effect and the specific heat of an electron gas, Phys. Rev. B 8, 2649 (1973).

[2] L. Zou and Y.-C. He, Field-induced $\mathrm{QCD}_{3}$-Chern-Simons quantum criticalities in Kitaev materials, Phys. Rev. Research 2, 013072 (2020).

[3] G. Baskaran and P. W. Anderson, Gauge theory of hightemperature superconductors and strongly correlated Fermi systems, Phys. Rev. B 37, 580 (1988).
[4] L. B. Ioffe and A. I. Larkin, Gapless fermions and gauge fields in dielectrics, Phys. Rev. B 39, 8988 (1989).

[5] P. A. Lee, Gauge Field, Aharonov-Bohm Flux, and High- $T_{c}$ Superconductivity, Phys. Rev. Lett. 63, 680 (1989).

[6] P. A. Lee and N. Nagaosa, Gauge theory of the normal state of high- $t_{c}$ superconductors, Phys. Rev. B 46, 5621 (1992).

[7] B. Blok and H. Monien, Gauge theories of high- $t_{c}$ superconductors, Phys. Rev. B 47, 3454 (1993). 
[8] M. U. Ubbens and P. A. Lee, Superconductivity phase diagram in the gauge-field description of the $t$-J model, Phys. Rev. B 49, 6853 (1994).

[9] C. Nayak and F. Wilczek, Non-Fermi liquid fixed point in $2+1$ dimensions, Nucl. Phys. B 417, 359 (1994).

[10] S. Chakravarty, R. E. Norton, and O. F. Syljuåsen, Transverse Gauge Interactions and the Vanquished Fermi Liquid, Phys. Rev. Lett. 74, 1423 (1995).

[11] M. Yu. Reizer, Relativistic effects in the electron density of states, specific heat, and the electron spectrum of normal metals, Phys. Rev. B 40, 11571 (1989).

[12] B. I. Halperin, P. A. Lee, and N. Read, Theory of the half-filled Landau level, Phys. Rev. B 47, 7312 (1993).

[13] J. Polchinski, Low-energy dynamics of the spinon-gauge system, Nucl. Phys. B 422, 617 (1994).

[14] B. L. Altshuler, L. B. Ioffe, and A. J. Millis, Low-energy properties of fermions with singular interactions, Phys. Rev. B 50, 14048 (1994).

[15] E.-A. Kim, M. J. Lawler, P. Oreto, S. Sachdev, E. Fradkin, and S. A. Kivelson, Theory of the nodal nematic quantum phase transition in superconductors, Phys. Rev. B 77, 184514 (2008).

[16] C. Nayak and F. Wilczek, Renormalization group approach to low temperature properties of a non-Fermi liquid metal, Nucl. Phys. B 430, 534 (1994).

[17] M. J. Lawler, D. G. Barci, V. Fernández, E. Fradkin, and L. Oxman, Nonperturbative behavior of the quantum phase transition to a nematic Fermi fluid, Phys. Rev. B 73, 085101 (2006).

[18] S.-S. Lee, Low-energy effective theory of Fermi surface coupled with $\mathrm{U}(1)$ gauge field in $2+1$ dimensions, Phys. Rev. B 80, 165102 (2009).

[19] M. A. Metlitski and S. Sachdev, Quantum phase transitions of metals in two spatial dimensions. I. Ising-nematic order, Phys. Rev. B 82, 075127 (2010).

[20] M. A. Metlitski and S. Sachdev, Quantum phase transitions of metals in two spatial dimensions. II. Spin density wave order, Phys. Rev. B 82, 075128 (2010).

[21] A. Abanov and A. Chubukov, Anomalous Scaling at the Quantum Critical Point in Itinerant Antiferromagnets, Phys. Rev. Lett. 93, 255702 (2004).

[22] A. Abanov and A. V. Chubukov, Spin-Fermion Model Near the Quantum Critical Point: One-Loop Renormalization Group Results, Phys. Rev. Lett. 84, 5608 (2000).

[23] D. F. Mross, J. McGreevy, H. Liu, and T. Senthil, Controlled expansion for certain non-Fermi-liquid metals, Phys. Rev. B 82, 045121 (2010).

[24] H.-C. Jiang, M. S. Block, R. V. Mishmash, J. R. Garrison, D. N. Sheng, O. I. Motrunich, and M. P. A. Fisher, Non-Fermi-liquid $d$-wave metal phase of strongly interacting electrons, Nature (London) 493, 39 (2013).

[25] S. B. Chung, I. Mandal, S. Raghu, and S. Chakravarty, Higher angular momentum pairing from transverse gauge interactions, Phys. Rev. B 88, 045127 (2013).

[26] Z. Wang, I. Mandal, S. B. Chung, and S. Chakravarty, Pairing in half-filled Landau level, Ann. Phys. 351, 727 (2014).

[27] S. Sur and S.-S. Lee, Chiral non-Fermi liquids, Phys. Rev. B 90, 045121 (2014).

[28] D. Dalidovich and S.-S. Lee, Perturbative non-Fermi liquids from dimensional regularization, Phys. Rev. B 88, 245106 (2013).
[29] S. Sur and S.-S. Lee, Quasilocal strange metal, Phys. Rev. B 91, 125136 (2015).

[30] I. Mandal and S.-S. Lee, Ultraviolet/infrared mixing in nonFermi liquids, Phys. Rev. B 92, 035141 (2015).

[31] I. Mandal, UV/IR Mixing in Non-Fermi Liquids: Higher-loop corrections in different energy ranges, Eur. Phys. J. B 89, 278 (2016).

[32] A. Eberlein, I. Mandal, and S. Sachdev, Hyperscaling violation at the Ising-nematic quantum critical point in two-dimensional metals, Phys. Rev. B 94, 045133 (2016).

[33] I. Mandal, Superconducting instability in non-Fermi liquids, Phys. Rev. B 94, 115138 (2016).

[34] I. Mandal, Scaling behaviour and superconducting instability in anisotropic non-Fermi liquids, Ann. Phys. 376, 89 (2017).

[35] A. Schlief, P. Lunts, and S.-S. Lee, Exact Critical Exponents for the Antiferromagnetic Quantum Critical Metal in Two Dimensions, Phys. Rev. X 7, 021010 (2017).

[36] P. Lunts, A. Schlief, and S.-S. Lee, Emergence of a control parameter for the antiferromagnetic quantum critical metal, Phys. Rev. B 95, 245109 (2017).

[37] S.-S. Lee, Recent developments in non-Fermi liquid theory, Annu. Rev. Condens. Matter Phys. 9, 227 (2018).

[38] D. Pimenov, I. Mandal, F. Piazza, and M. Punk, Non-Fermi liquid at the FFLO quantum critical point, Phys. Rev. B 98 , 024510 (2018).

[39] A. A. Abrikosov, Calculation of critical indices for zero-gap semiconductors, JETP 39, 709 (1974).

[40] E.-G. Moon, C. Xu, Y. B. Kim, and L. Balents, NonFermi-Liquid and Topological States with Strong Spin-Orbit Coupling, Phys. Rev. Lett. 111, 206401 (2013).

[41] R. M. Nandkishore and S. A. Parameswaran, Disorderdriven destruction of a non-Fermi liquid semimetal studied by renormalization group analysis, Phys. Rev. B 95, 205106 (2017).

[42] I. Mandal and R. M. Nandkishore, Interplay of Coulomb interactions and disorder in three-dimensional quadratic band crossings without time-reversal symmetry and with unequal masses for conduction and valence bands, Phys. Rev. B 97, 125121 (2018).

[43] O. I. Motrunich, Variational study of triangular lattice spin$1 / 2$ model with ring exchanges and spin liquid state in $\kappa$-(ET) $)_{2} \mathrm{Cu}_{2}(\mathrm{CN})_{3}$, Phys. Rev. B 72, 045105 (2005).

[44] S.-S. Lee and P. A. Lee, U(1) Gauge Theory of the Hubbard Model: Spin Liquid States and Possible Application to $\kappa$-(BEDT-TTF $)_{2} \mathrm{Cu}_{2}(\mathrm{CN})_{3}$, Phys. Rev. Lett. 95, 036403 (2005).

[45] P. A. Lee, N. Nagaosa, and X.-G. Wen, Doping a Mott insulator: Physics of high-temperature superconductivity, Rev. Mod. Phys. 78, 17 (2006).

[46] O. I. Motrunich and M. P. A. Fisher, $d$-wave correlated critical Bose liquids in two dimensions, Phys. Rev. B 75, 235116 (2007).

[47] V. Oganesyan, S. A. Kivelson, and E. Fradkin, Quantum theory of a nematic Fermi fluid, Phys. Rev. B 64, 195109 (2001).

[48] W. Metzner, D. Rohe, and S. Andergassen, Soft Fermi Surfaces and Breakdown of Fermi-Liquid Behavior, Phys. Rev. Lett. 91, 066402 (2003).

[49] L. Dell'Anna and W. Metzner, Fermi surface fluctuations and single electron excitations near Pomeranchuk instability in two dimensions, Phys. Rev. B 73, 045127 (2006). 
[50] H.-Y. Kee, E. H. Kim, and C.-H. Chung, Signatures of an electronic nematic phase at the isotropic-nematic phase transition, Phys. Rev. B 68, 245109 (2003).

[51] J. Rech, C. Pépin, and A. V. Chubukov, Quantum critical behavior in itinerant electron systems: Eliashberg theory and instability of a ferromagnetic quantum critical point, Phys. Rev. B 74, 195126 (2006).

[52] P. Wölfle and A. Rosch, Fermi liquid near a quantum critical point, J. Low Temp. Phys. 147, 165 (2007).

[53] D. L. Maslov and A. V. Chubukov, Fermi liquid near Pomeranchuk quantum criticality, Phys. Rev. B 81, 045110 (2010).

[54] J. Quintanilla and A. J. Schofield, Pomeranchuk and topological Fermi surface instabilities from central interactions, Phys. Rev. B 74, 115126 (2006).

[55] H. Yamase and H. Kohno, Instability toward formation of quasione-dimensional Fermi surface in two-dimensional $t$ - $J$ model, J. Phys. Soc. Jpn. 69, 2151 (2000).

[56] H. Yamase, V. Oganesyan, and W. Metzner, Mean-field theory for symmetry-breaking Fermi surface deformations on a square lattice, Phys. Rev. B 72, 035114 (2005).

[57] C. J. Halboth and W. Metzner, $d$-Wave Superconductivity and Pomeranchuk Instability in the Two-Dimensional Hubbard Model, Phys. Rev. Lett. 85, 5162 (2000).

[58] P. Jakubczyk, P. Strack, A. A. Katanin, and W. Metzner, Renormalization group for phases with broken discrete symmetry near quantum critical points, Phys. Rev. B 77, 195120 (2008).

[59] M. Zacharias, P. Wölfle, and M. Garst, Multiscale quantum criticality: Pomeranchuk instability in isotropic metals, Phys. Rev. B 80, 165116 (2009).
[60] Y. Huh and S. Sachdev, Renormalization group theory of nematic ordering in $d$-wave superconductors, Phys. Rev. B 78, 064512 (2008).

[61] T. Senthil and R. Shankar, Fermi Surfaces in General Codimension and a New Controlled Nontrivial Fixed Point, Phys. Rev. Lett. 102, 046406 (2009).

[62] L. Zou and D. Chowdhury, Deconfined metallic quantum criticality: A $u$ (2) gauge-theoretic approach, Phys. Rev. Research 2, 023344 (2020).

[63] The $k_{F}$-dependence drops out for $m=1$.

[64] S.-S. Lee, Stability of the U(1) spin liquid with a spinon Fermi surface in 2+1 dimensions, Phys. Rev. B 78, 085129 (2008).

[65] S. Sachdev, Quantum Phase Transitions, 2nd ed. (Cambridge University Press, Cambridge, 2011).

[66] B. Keimer, S. A. Kivelson, M. R. Norman, S. Uchida, and J. Zaanen, From quantum matter to high-temperature superconductivity in copper oxides, Nature (London) 518, 179 (2015).

[67] T. Senthil, S. Sachdev, and M. Vojta, Fractionalized Fermi Liquids, Phys. Rev. Lett. 90, 216403 (2003).

[68] While performing the integral for $k_{d-m+1}$, we can neglect the contribution from the exponential term as $k_{d-m+1}$ already appears in the denominator and is appropriately damped. For extracting leading order singular behavior in $k_{F}$, this is sufficient.

Correction: The first sentence of the Introduction, Eqs. (1), (11), and (13), and text after Eqs. (4) and (43) contained minor errors and have been fixed. 